\title{
Fast and Accurate Trajectory Tracking Control of an Autonomous Surface Vehicle with Unmodelled Dynamics and Disturbances
}

\author{
Ning Wang, Senior Member, IEEE, Shuailin Lv, Meng Joo Er, Senior Member, IEEE \\ and Wen-Hua Chen, Senior Member, IEEE
}

\begin{abstract}
In this paper, fast and accurate trajectory tracking control of an autonomous surface vehicle (ASV) with complex unknowns including unmodeled dynamics, uncertainties and/or unknown disturbances is addressed within a proposed homogeneity-based finite-time control (HFC) framework. Major contributions are as follows: (1) In the absence of external disturbances, a nominal HFC framework is established to achieve exact trajectory tracking control of an ASV, whereby global finitetime stability is ensured by combining homogeneous analysis and Lyapunov approach; (2) Within the HFC scheme, a finite-time disturbance observer (FDO) is further nested to rapidly and accurately reject complex disturbances, and thereby contributing to an FDO-based HFC (FDO-HFC) scheme which can realize exactness of trajectory tracking and disturbance observation; (3) Aiming to exactly deal with complicated unknowns including unmodeled dynamics and/or disturbances, a finite-time unknown observer (FUO) is deployed as a patch for the nominal HFC framework, and eventually results in an FUO-based HFC (FUOHFC) scheme which guarantees that accurate trajectory tracking can be achieved for an ASV under harsh environments. Simulation studies and comprehensive comparisons conducted on a benchmark ship demonstrate the effectiveness and superiority of the proposed HFC schemes.
\end{abstract}

Index Terms-Global finite-time stability, accurate trajectory tracking, finite-time disturbance observer (FDO), finite-time unknown observer (FUO), autonomous surface vehicle (ASV).

\section{INTRODUCTION}

$\mathbf{I}$ N RECENT YEARS, autonomous surface vehicles (ASVs) have been widely deployed for various missions related with observations, military tasks, coastal and inland waters monitoring, etc., [1]. Generally, tracking control of an ASV to a prescribed trajectory/path with an acceptable accuracy plays a key role within the entire autopilot system and has thus attracted great attention from both marine engineering and control communities [2]. It is much more demanding to achieve accurate tracking of a pre-determined trajectory in

Manuscript received October 17, 2016; revised January 20, 2017. This work is supported by the National Natural Science Foundation of P. R. China (under Grants 51009017 and 51379002), the Fund for Dalian Distinguished Young Scholars (under Grant 2016RJ10), the Innovation Support Plan for Dalian High-level Talents (under Grant 2015R065), and the Fundamental Research Funds for the Central Universities (under Grant 3132016314).

N. Wang and S. Lv are with the Marine Engineering College, Dalian Maritime University, Dalian 116026, China. (email: \{n.wang.dmu.cn, lvshuailin\}@gmail.com).

M. Er is with the School of Electrical and Electronic Engineering, Nanyang Technological University, Singapore. (e-mail: emjer@ntu.edu.sg).

W.-H. Chen is with the Department of Aeronautical and Automotive Engineering, Loughborough University, Loughborough, LE11 3TU, U.K. (email:w.chen@lboro.ac.uk). some applications; for example, marine surveying and mapping on the sea in the presence of complicated uncertainties and variations including system uncertainties and external disturbances due to ocean winds, waves and currents [3]-[7]. In this context, it becomes extremely challenging to achieve accurate trajectory tracking of an ASV sailing in such harsh environments.

The sliding mode control (SMC) technique has been investigated as a promising approach to achieve high accurate trajectory tracking control of an ASV [8]. However, the SMCbased approaches have to incur high-frequency chattering with conservatively large magnitude around the sliding surface to dominate unknowns and achieve the robustness. Furthermore, the SMC technique can only handle matched unknowns. By virtue of the (vectorial) backstepping technique [9], a trajectory tracking controller has been designed for an ASV in the presence of (mismatched) unknowns including time-varying disturbances and system uncertainties. It should be noted that tracking errors can only be made globally uniformly ultimately bounded. Applying the integrator backstepping [10] technique to the design of trajectory tracking control law for an underactuated ASV contributes to semi-globally exponentially stable tracking errors. Unfortunately, uncertainties and disturbances have not been addressed. In combination with neural networks (NNs) and adaptive robust control techniques [11], a saturated tracking controller that renders tracking errors semi-globally uniformly ultimately bounded has been proposed to preserve the robustness against time-varying disturbances induced by waves and ocean currents. In addition to NNs [12]-[14], a lot of efforts on adaptive approximation based tracking control have also been made via fuzzy systems (FS) [15]-[19], and fuzzy neural networks (FNN) [20]-[25], etc., and can roughly compensate unknown dynamics. Recently, a significant progress has been made by an innovative approximator termed self-constructing fuzzy neural network (SCFNN) [26]-[29] towards the dynamic-structure-approximation based adaptive control approaches [20] with much higher accuracy of both reconstruction and trajectory tracking. It should be highlighted that accurate tracking control can still hardly be achieved by the foregoing SCFNN-based control approaches since there still exist unexpected approximation residuals. Nevertheless, the convergence rate of tracking errors is usually somewhat slow since only asymptotic or exponential closed-loop stability can be derived from previous tracking control approaches.

In order to further pursue better tracking performance, 
finite-time control approaches have been implemented in the literature [30], [31]. In [30], the SMC technique has been employed to realize attitude tracking of a rigid spacecraft and a modified differentiator has been incorporated to compensate disturbances and inertia uncertainties, whereby finitetime convergence of tracking errors can be obtained. By virtue of the homogeneous method in [31], finite-time stability of the closed-loop control system with negative degree of homogeneous can be ensured if tracking error dynamics can be proved to be asymptotically stable. It should be noted that, compared with traditional asymptotic convergent methods, the aforementioned finite-time control approaches can achieve not only faster convergence rate within the vicinity of the origin but also stronger disturbance rejection. Meaningfully, finitetime control approaches ensure that tracking errors can reach to zero within a finite time. Note that, unlike SMC-based approaches, the homogeneity-based method [31] contributes to a straightforward solution without any couplings of tracking errors. Besides, external disturbances can even excite unmodeled dynamics of the ASV, and thus require to be well estimated. Otherwise, complex disturbances pertaining to an ASV cannot be exactly observed within a short time, and make trajectory tracking inaccurate.

Recently, disturbance observer based control (DOBC) technique has also been proposed by Chen [32] to not only improve system robustness but also enhance the entire performance without sacrificing the nominal one [33]-[35]. In this context, the DOBC schemes have been extensively studied and widely applied to various industrial sectors, including mechatronics systems [36], aerospace systems [37], and process control systems [38]. Clearly, it is innovative within the DOBC framework that all disturbances and/or unknowns are addressed as a lumped nonlinearity estimated by a nonlinear disturbance observer (NDO) which is usually involved.

In this paper, an ambitious goal of achieving fast and accurate trajectory control of an ASV in the presence of unknowns including disturbances and unmodelled dynamics is pursued. To be specific, a homogeneity-based finite-time control (HFC) scheme is developed to achieve accurate trajectory tracking of an ASV in the absence of external disturbances. In conjunction with a finite-time disturbance observer (FDO) which can be further devised to exactly estimate external disturbances within a short time, a FDO-based HFC (FDO-HFC) scheme is thus implemented to exactly track an ASV suffering from complex disturbances. In order to further address unmodelled dynamics including uncertainties and/or excited dynamics due to disturbances, a high-order sliding mode estimator is designed to realize a finite-time unknown observer (FUO) which can exactly capture unknown dynamics. Incorporating the FUO into the HFC scheme contributes to the FUO-based HFC (FUO-HFC) scheme which can achieve fast and accurate trajectory tracking with complex unknowns including unmodelled dynamics and/or uncertainties in addition to disturbances.

The rest of this paper is organized as follows. In Section II, preliminaries together with the trajectory tracking problem associated with an ASV are addressed. The HFC, FDO-HFC and FUO-HFC schemes together with theoretical analysis on finite-time stability are presented in Section III. Simulation studies and discussions are conducted in Section IV. Conclusions are drawn in Section V.

\section{Preliminaries and Problem Statement}

\section{A. Preliminaries}

For the convenience of readers, we collect the key definitions and lemmas frequently used in this paper in the sequel.

Consider an autonomous nonlinear system as follows:

$\dot{\boldsymbol{x}}(t)=\boldsymbol{f}(\boldsymbol{x}(t)), \quad x(0)=0, \quad \boldsymbol{f}(0)=0, \quad \boldsymbol{x} \in U_{0} \subset \mathbb{R}^{n}$

where $x=\left[x_{1}, \cdots, x_{n}\right]^{T}$ and nonlinear function $\boldsymbol{f}(\cdot)$ is continuous on a open neighborhood $U_{0}$ of the origin.

Definition 1 (Globally Asymptotic Stability [39]): The equilibrium $x_{e}=0$ of system (1) is globally asymptotically stable if there exits a function $V(\boldsymbol{x})$ satisfying

(a) $V(0)=0$;

(b) $V(\boldsymbol{x})>0, \forall \boldsymbol{x} \neq 0$, and $V(\boldsymbol{x})$ is radically unbounded;

(c) $\dot{V}(\boldsymbol{x}) \leq 0$;

(d) $\dot{V}(\boldsymbol{x})$ does not vanish identically along any trajectory in $\mathbb{R}^{n}$, other than the null solution $x=0$.

Definition 2 (Homogeneity [31]): Denote $V(\boldsymbol{x}): \mathbb{R}^{n} \rightarrow$ $\mathbb{R}$ be a continuous scalar function. $V(\boldsymbol{x})$ is said to be a homogeneous function of degree $\sigma$ with respect to weights $\left(r_{1}, \cdots, r_{n}\right) \in \mathbb{R}^{n}$ with $r_{i}>0, i=1,2, \cdots, n$, if, for any given $\varepsilon>0$,

$V\left(\varepsilon^{r_{1}} x_{1}, \cdots, \varepsilon^{x_{n}} x_{n}\right)=\varepsilon^{\sigma} V(\boldsymbol{x}), \quad i=1, \cdots, n, \forall \boldsymbol{x} \in \mathbb{R}^{n}$.

Denote $\boldsymbol{f}(\boldsymbol{x})=\left[f_{1}(\boldsymbol{x}), \cdots, f_{n}(\boldsymbol{x})\right]^{T}$ be a continuous vector field. $\boldsymbol{f}(\boldsymbol{x})$ is homogeneous of degree $k \in \mathbb{R}$ with respect to weights $\left(r_{1}, \cdots, r_{n}\right)$, if, for any given $\varepsilon>0$,

$f_{i}\left(\varepsilon^{r_{1}} x_{1}, \cdots, \varepsilon^{r_{n}} x_{n}\right)=\varepsilon^{k+r_{i}} f_{i}(\boldsymbol{x}), \quad i=1, \cdots, n, \forall \boldsymbol{x} \in \mathbb{R}^{n}$.

And, system (1) is said to be homogeneous if $\boldsymbol{f}(\boldsymbol{x})$ is homogeneous.

In combination with Definitions 1 and 2, a fundamental result on global finite-time stability can be obtained as follows:

Lemma 1 (Global Finite-Time Stability [31]): System (1) is globally finite-time stable if system (1) is globally asymptotically stable and is homogeneous of a negative degree.

By virtue of Lemma 1, we can derive a cornerstone result, whose proof is presented in details in Appendix A, for finitetime observer design and analysis in this paper.

Lemma 2: The following system:

$$
\begin{aligned}
& \dot{z}_{1}=z_{2}-l_{1} \operatorname{sig}^{\alpha_{2}}\left(z_{1}\right) \\
& \dot{z}_{2}=z_{3}-l_{2} \operatorname{sig}^{\alpha_{3}}\left(z_{1}\right) \\
& \quad \vdots \\
& \dot{z}_{n}=-l_{n} \operatorname{sig}^{\alpha_{n+1}}\left(z_{1}\right)
\end{aligned}
$$

where $l_{i}>0, i=1,2, \cdots, n$ are appropriate constants, and

$$
\operatorname{sig}^{\alpha_{i}}\left(z_{1}\right):=\left|z_{1}\right|^{\alpha_{i}} \operatorname{sgn}\left(z_{1}\right)
$$

with $\alpha_{i+1}=\alpha_{i}+\tau, i=1,2, \cdots, n$ and $\alpha_{1}=1$ for any $\tau<0$, is globally finite-time stable. 


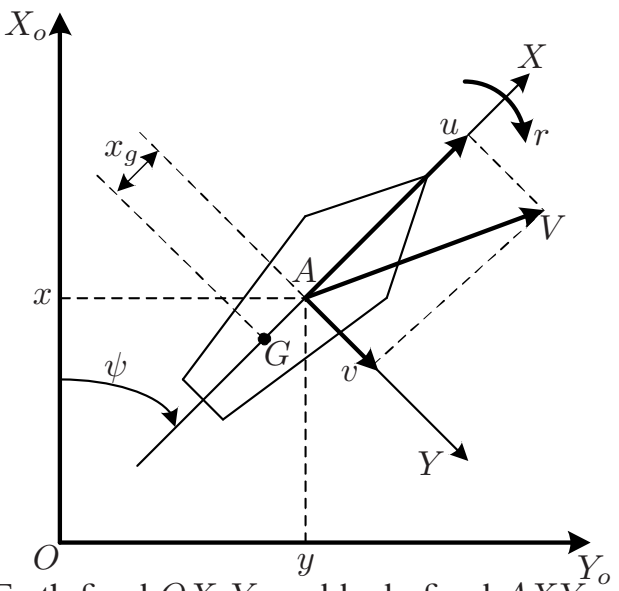

Fig. 1: Earth-fixed $O X_{o} Y_{o}$ and body-fixed $A X Y^{\circ}$ coordinate frames of an ASV

\section{B. Problem Formulation}

Let $\boldsymbol{\eta}=[x, y, \psi]^{T}$ denote the 3-DOF position $(x, y)$ and heading angle $(\psi)$ of the ASV in the earth-fixed inertial frame as shown in Fig. 1, and let $\boldsymbol{\nu}=[u, v, r]^{T}$ denote the corresponding linear velocities $(u, v)$, i.e., surge and sway velocities, and angular rate $(r)$, i.e., yaw, in the body-fixed frame. An ASV sailing in a planar space can be modeled as follows [3]:

$$
\begin{aligned}
\dot{\boldsymbol{\eta}} & =\mathbf{R}(\psi) \boldsymbol{\nu} \\
\mathbf{M} \dot{\boldsymbol{\nu}} & =\boldsymbol{f}(\boldsymbol{\eta}, \boldsymbol{\nu})+\boldsymbol{\tau}+\boldsymbol{\tau}_{d}
\end{aligned}
$$

with dynamics $\boldsymbol{f}(\boldsymbol{\eta}, \boldsymbol{\nu})$ usually modeled by

$$
\boldsymbol{f}(\boldsymbol{\eta}, \boldsymbol{\nu})=-\mathbf{C}(\boldsymbol{\nu}) \boldsymbol{\nu}-\mathbf{D}(\boldsymbol{\nu}) \boldsymbol{\nu}-\boldsymbol{g}(\boldsymbol{\eta}, \boldsymbol{\nu})
$$

where $\boldsymbol{\tau}=\left[\tau_{1}, \tau_{2}, \tau_{3}\right]^{T}$ and $\tau_{d}:=\mathbf{M R}^{T}(\psi) \boldsymbol{d}(t)$ with $\boldsymbol{d}(t)=\left[d_{1}(t), d_{2}(t), d_{3}(t)\right]^{T}$ are control input and mixed disturbances, respectively, and $g$ denote the restoring forces and moments due to gravitation/buoyancy. The term $\mathbf{R}(\psi)$ is a rotation matrix given by

$$
\mathbf{R}(\psi)=\left[\begin{array}{ccc}
\cos \psi & -\sin \psi & 0 \\
\sin \psi & \cos \psi & 0 \\
0 & 0 & 1
\end{array}\right]
$$

with the following properties:

$$
\begin{gathered}
\mathbf{R}^{T}(\psi) \mathbf{R}(\psi)=\mathbf{I}, \text { and }\|\mathbf{R}(\psi)\|=1, \forall \psi \in[0,2 \pi] \\
\dot{\mathbf{R}}(\psi)=\mathbf{R}(\psi) \mathbf{S}(r) \\
\mathbf{R}^{T}(\psi) \mathbf{S}(r) \mathbf{R}(\psi)=\mathbf{R}(\psi) \mathbf{S}(r) \mathbf{R}^{T}(\psi)=\mathbf{S}(r)
\end{gathered}
$$

where $\mathbf{S}(r)=\left[\begin{array}{ccc}0 & -r & 0 \\ r & 0 & 0 \\ 0 & 0 & 0\end{array}\right]$, the inertia matrix $\mathbf{M}=$ $\mathbf{M}^{T}>0$, the skew-symmetric matrix $\mathbf{C}(\boldsymbol{\nu})=-\mathbf{C}(\boldsymbol{\nu})^{T}$ and the damping matrix $\mathbf{D}(\boldsymbol{\nu})$ are given by

$$
\begin{aligned}
\mathbf{M}= & {\left[\begin{array}{ccc}
m_{11} & 0 & 0 \\
0 & m_{22} & m_{23} \\
0 & m_{32} & m_{33}
\end{array}\right] } \\
\mathbf{C}(\boldsymbol{\nu})= & {\left[\begin{array}{ccc}
0 & 0 & c_{13}(\boldsymbol{\nu}) \\
0 & 0 & c_{23}(\boldsymbol{\nu}) \\
-c_{13}(\boldsymbol{\nu}) & -c_{23}(\boldsymbol{\nu}) & 0
\end{array}\right] } \\
\mathbf{D}(\boldsymbol{\nu})= & {\left[\begin{array}{ccc}
d_{11}(\boldsymbol{\nu}) & 0 & 0 \\
0 & d_{22}(\boldsymbol{\nu}) & d_{23}(\boldsymbol{\nu}) \\
0 & d_{32}(\boldsymbol{\nu}) & d_{33}(\boldsymbol{\nu})
\end{array}\right] }
\end{aligned}
$$

where $m_{11}=m-X_{\dot{u}}, m_{22}=m-Y_{\dot{v}}, m_{23}=m x_{g}-$ $Y_{\dot{r}}, m_{32}=m x_{g}-N_{\dot{v}}, m_{33}=I_{z}-N_{\dot{r}} ; c_{13}(\boldsymbol{\nu})=$ $-m_{11} v-m_{23} r, \quad c_{23}(\boldsymbol{\nu})=m_{11} u ; \quad d_{11}(\boldsymbol{\nu})=-X_{u}-$ $X_{|u| u}|u|-X_{\text {uиu }} u^{2}, d_{22}(\boldsymbol{\nu})=-Y_{v}-Y_{|v| v \mid}|v|, \quad d_{23}(\boldsymbol{\nu})=$ $-Y_{r}-Y_{|v| r}|v|-Y_{|r| r}|r|, d_{32}(\boldsymbol{\nu})=-N_{v}-N_{|v| v}|v|-N_{|r| v}|r|$ and $d_{33}(\boldsymbol{\nu})=-N_{r}-N_{|v| r}|v|-N_{|r| r \mid}|r|$. Here, $m$ is the mass of the vessel, $I_{z}$ is the moment of inertia about the yaw rotation, $Y_{\dot{r}}=N_{\dot{v}}$, and $X_{*}, Y_{*}$ and $N_{*}$ denote corresponding hydrodynamic derivatives which are actually difficult to be accurately obtained.

Consider the following desired trajectory:

$$
\begin{aligned}
\dot{\boldsymbol{\eta}}_{d} & =\mathbf{R}\left(\psi_{d}\right) \boldsymbol{\nu}_{d} \\
\mathbf{M} \dot{\boldsymbol{\nu}}_{d} & =\boldsymbol{f}_{0}\left(\boldsymbol{\eta}_{d}, \boldsymbol{\nu}_{d}\right)
\end{aligned}
$$

where $\boldsymbol{f}_{0}(\cdot)$ is the nominal dynamics, $\boldsymbol{\eta}_{d}=\left[x_{d}, y_{d}, \psi_{d}\right]^{T}$ and $\boldsymbol{\nu}_{d}=\left[u_{d}, v_{d}, r_{d}\right]^{T}$ are the desired position and velocity vectors, respectively.

In this context, the control objective is to design a controller $\boldsymbol{\tau}$ such that the actual position and velocity vectors (i.e., $\boldsymbol{\eta}$ and $\boldsymbol{\nu})$ of the ASV in (4) can track exactly the desired trajectory (i.e., $\boldsymbol{\eta}_{d}$ and $\boldsymbol{\nu}_{d}$ ) generated by (9) in a finite time.

Remark 1: Clearly, in addition to unknown disturbances $\tau_{d}$, if the dynamics $f$ of the ASV in (4) cannot be sufficiently modeled due to parametric unknowns including $\mathbf{C}, \mathbf{D}$ and $\boldsymbol{g}$, and/or structural unmodeled dynamics, accurate trajectory tracking control of an ASV under harsh environments would become extremely challenging.

\section{Homogeneity-BASEd Finite-Time Tracking CONTROL SCHEME}

\section{A. Nominal Homogeneity-Based Finite-Time Contol}

In order to facilitate the controller design and analysis, we introduce an auxiliary velocity vector as follows:

$$
\begin{aligned}
\boldsymbol{w} & =\mathbf{R} \boldsymbol{\nu} \\
\boldsymbol{w}_{d} & =\mathbf{R}\left(\psi_{d}\right) \boldsymbol{\nu}_{d}
\end{aligned}
$$

where $\boldsymbol{w}=\left[w_{1}, w_{2}, w_{3}\right]^{T}, \boldsymbol{w}_{d}=\left[w_{d, 1}, w_{d, 2}, w_{d, 3}\right]^{T}, \mathbf{R}=$ $\mathbf{R}(\psi)$ and $\mathbf{R}_{d}=\mathbf{R}\left(\psi_{d}\right)$.

Together with (4) and (10a), using properties in (7), we have

$$
\begin{aligned}
\dot{\boldsymbol{\eta}} & =\boldsymbol{w} \\
\dot{\boldsymbol{w}} & =\mathbf{R M}^{-1} \boldsymbol{\tau}+\boldsymbol{h}(\boldsymbol{\eta}, \boldsymbol{w})+\boldsymbol{d}(t)
\end{aligned}
$$

where

$$
\boldsymbol{h}(\boldsymbol{\eta}, \boldsymbol{w})=\mathbf{S}\left(w_{3}\right) \boldsymbol{w}+\mathbf{R M}^{-1} \boldsymbol{f}\left(\boldsymbol{\eta}, \mathbf{R}^{T} \boldsymbol{w}\right)
$$


Similarly, using (9) and (10b), we have

$$
\begin{aligned}
\dot{\boldsymbol{\eta}}_{d} & =\boldsymbol{w}_{d} \\
\dot{\boldsymbol{w}}_{d} & =\mathbf{S}\left(w_{d, 3}\right) \boldsymbol{w}_{d}+\mathbf{R}_{d} \mathbf{M}^{-1} \boldsymbol{f}_{0}\left(\boldsymbol{\eta}_{d}, \mathbf{R}_{d}^{T} \boldsymbol{w}_{d}\right)
\end{aligned}
$$

Combining with (11) and (13), we have

$$
\begin{aligned}
\dot{\boldsymbol{\eta}}_{e} & =\boldsymbol{w}_{e} \\
\dot{\boldsymbol{w}}_{e} & =\mathbf{R M}^{-1} \boldsymbol{\tau}+\boldsymbol{h}_{e}\left(\boldsymbol{\eta}, \boldsymbol{w}, \boldsymbol{\eta}_{d}, \boldsymbol{w}_{d}\right)+\boldsymbol{d}(t)
\end{aligned}
$$

where $\boldsymbol{\eta}_{e}=\boldsymbol{\eta}-\boldsymbol{\eta}_{d}:=\left[\eta_{e, 1}, \eta_{e, 2}, \eta_{e, 3}\right]^{T}, \boldsymbol{w}_{e}=\boldsymbol{w}-\boldsymbol{w}_{d}:=$ $\left[w_{e, 1}, w_{e, 2}, w_{e, 3}\right]^{T}$, and

$$
\begin{aligned}
\boldsymbol{h}_{e}\left(\boldsymbol{\eta}, \boldsymbol{w}, \boldsymbol{\eta}_{d}, \boldsymbol{w}_{d}\right)= & \mathbf{S} \boldsymbol{w}-\mathbf{S}_{d} \boldsymbol{w}_{d}+\mathbf{R} \mathbf{M}^{-1} \boldsymbol{f}\left(\boldsymbol{\eta}, \mathbf{R}^{T} \boldsymbol{w}\right) \\
& -\mathbf{R}_{d} \mathbf{M}^{-1} \boldsymbol{f}_{0}\left(\boldsymbol{\eta}_{d}, \mathbf{R}_{d}^{T} \boldsymbol{w}_{d}\right)
\end{aligned}
$$

with $\mathbf{S}=\mathbf{S}\left(w_{3}\right)$ and $\mathbf{S}_{d}=\mathbf{S}\left(w_{d, 3}\right)$.

Starting from the tracking error dynamics (14), we set out to design a nominal homogeneity-based finite-time control (HFC) scheme which is expected to ensure that the tracking errors $\boldsymbol{\eta}_{e}$ and $\boldsymbol{w}_{e}$ converge to zero in a finite time.

To this end, a nominal HFC scheme for the ASV in (4) without disturbances (i.e., $\boldsymbol{\tau}_{d}=0$ or $\boldsymbol{d}(t)=0$ ) is developed by employing the homogeneous theory. Moreover, we show that finite-time stability of the entire closed-loop tracking system can be ensured by using the Lyapunov synthesis.

Design the nominal HFC law $\tau_{\mathrm{HFC}}$ as follows:

$$
\begin{aligned}
\boldsymbol{\tau}_{\mathrm{HFC}}= & -\mathbf{M R} \mathbf{R}^{-1}\left(K_{1} \operatorname{sig}^{\beta_{1}}\left(\boldsymbol{\eta}-\boldsymbol{\eta}_{d}\right)+K_{2} \operatorname{sig}^{\beta_{2}}\left(\mathbf{R} \boldsymbol{\nu}-\mathbf{R}_{d} \boldsymbol{\nu}_{d}\right)\right) \\
& -\mathbf{M S} \boldsymbol{\nu}+\mathbf{M R}^{-1} \mathbf{S}_{d} \mathbf{R}_{d} \boldsymbol{\nu}_{d} \\
& -\boldsymbol{f}(\boldsymbol{\eta}, \boldsymbol{\nu})+\mathbf{M R}^{-1} \mathbf{R}_{d} \mathbf{M}^{-1} \boldsymbol{f}_{0}\left(\boldsymbol{\eta}_{d}, \boldsymbol{\nu}_{d}\right)
\end{aligned}
$$

where $\operatorname{sig}^{\beta_{i}}(\boldsymbol{x})=\left[\operatorname{sig}^{\beta_{i}}\left(x_{1}\right), \cdots, \operatorname{sig}^{\beta_{i}}\left(x_{n}\right)\right]^{T}, i=1,2$, $K_{1}>0, K_{2}>0,0<\beta_{1}<1$ and $\beta_{2}=2 \beta_{1} /\left(1+\beta_{1}\right)$.

It is essential that the proposed HFC scheme can make the ASV in (4) track exactly the desired trajectory generated by (9) in a finite time. The key result ensuring the closed-loop finite-time stability is now stated.

Theorem 1 (HFC): Using the HFC scheme governed by (16), the ASV in (4) can exactly track the desired trajectory generated by (9) within a finite time $0<T<\infty$, i.e., $\boldsymbol{\eta}(t) \equiv$ $\boldsymbol{\eta}_{d}(t), \boldsymbol{\nu}(t) \equiv \boldsymbol{\nu}_{d}(t), \forall t \geq T$.

Proof: Substituting the HFC law (16) into the tracking error system (14) without considering disturbances yields the closed-loop tracking error dynamics as follows:

$$
\begin{aligned}
\dot{\eta}_{e, j} & =w_{e, j} \\
\dot{w}_{e, j} & =-K_{1} \operatorname{sig}^{\beta_{1}}\left(\eta_{e, j}\right)-K_{2} \operatorname{sig}^{\beta_{2}}\left(w_{e, j}\right)
\end{aligned}
$$

for $j=1,2,3$.

In light of Lemma 1, global asymptotic stability and negative homogeneity of system (17) are expected to be guaranteed respectively in the sequel.

1) Global Asymptotic Stability: Consider the following Lyapunov function:

$$
V\left(\boldsymbol{\eta}_{e}, \boldsymbol{w}_{e}\right)=\sum_{j=1}^{3}\left(K_{1} \int_{0}^{\eta_{e, j}} \operatorname{sig}^{\beta_{1}}(\mu) d \mu+\frac{1}{2} w_{e, j}^{2}\right)
$$

Differentiating $V\left(\boldsymbol{\eta}_{e}, \boldsymbol{w}_{e}\right)$ along the tracking error dynamics (17), we have

$$
\begin{aligned}
\dot{V}\left(\boldsymbol{\eta}_{e}, \boldsymbol{w}_{e}\right)= & K_{1} \sum_{j=1}^{3} \operatorname{sig}^{\beta_{1}}\left(\eta_{e, j}\right) w_{e, j} \\
& -\sum_{j=1}^{3} w_{e, j}\left(K_{1} \operatorname{sig}^{\beta_{1}}\left(\eta_{e, j}\right)+K_{2} \operatorname{sig}^{\beta_{2}}\left(w_{e, j}\right)\right) \\
= & -K_{2} \sum_{j=1}^{3} w_{e, j} \operatorname{sig}^{\beta_{2}}\left(w_{e, j}\right) \\
= & -K_{2} \sum_{j=1}^{3}\left|w_{e, j}\right|^{1+\beta_{2}}
\end{aligned}
$$

which yields $V(t)$ is bounded as time $t$ tends to infinity, i.e.,

$$
\frac{1}{2}\left\|\boldsymbol{w}_{e}(t)\right\|^{2} \leq V(t)<\infty
$$

Using $\left\|\boldsymbol{w}_{e}(t)\right\|^{1+\beta_{2}} \leq\left\|\boldsymbol{w}_{e}(t)\right\|^{2}+1$, we further have

$$
\left\|\boldsymbol{w}_{e}^{\left(1+\beta_{2}\right) / 2}(t)\right\| \leq \sqrt{2 V(t)+1}<\infty
$$

Note, from (19), that

$$
\int_{0}^{t}\left\|\boldsymbol{w}_{e}^{\left(1+\beta_{2}\right) / 2}(\tau)\right\|^{2} d \tau=\frac{V(0)-V(t)}{K_{2}}<\infty
$$

Combining with (21) and (22) and using Barbalat's lemma [40] yields

$$
\lim _{t \rightarrow \infty} w_{e}(t)=0
$$

In what follows, we expect to prove that $\boldsymbol{\eta}_{e}(t)$ also converges to zero as time $t$ tends to infinity. To this end, a proof by contradiction is employed by assuming that $\boldsymbol{\eta}_{e}(t)$ converges to a nonzero constant $\boldsymbol{\eta}_{0} \neq 0$.

Together with (17) and (23), we have, as $t \rightarrow \infty$,

$$
\begin{aligned}
\dot{\eta}_{e, j} & =0 \\
\dot{w}_{e, j} & =-K_{1} \operatorname{sig}^{\beta_{1}}\left(\eta_{e, j}\right)
\end{aligned}
$$

which, combining with the hypothesis, implies that $\eta_{e, j}$ converges to a nonzero constant $\eta_{0, j} \neq 0$, and thereby $\dot{w}_{e, j}=$ $-K_{1} \operatorname{sig}^{\beta_{1}}\left(\eta_{e, j}\right) \neq 0$. In this context, $w_{e, j}$ deviates from the origin and makes $\dot{\eta}_{e, j} \neq 0$, and thereby resulting in a new convergent constant $\bar{\eta}_{0, j}$ different from the assumed one, i.e., $\bar{\eta}_{0, j} \neq \eta_{0, j}$. This leads to a contradiction and thus yields

$$
\lim _{t \rightarrow \infty} \boldsymbol{\eta}_{e}(t)=0
$$

It follows from (23) and (25) that using the HFC law in (16), system (14) without disturbances (i.e., $\boldsymbol{d}(t)=0$ ) is globally asymptotically stable.

2) Negative Homogeneity: For system (17), selecting a dilation as follows:

$$
\left(r_{1}, r_{2}\right)=\left(1, \frac{1+\beta_{1}}{2}\right)
$$

for any given $\varepsilon>0$, yields

$$
\begin{aligned}
& f_{1}\left(\varepsilon^{r_{1}} \eta_{e, j}, \varepsilon^{r_{2}} w_{e, j}\right)=\varepsilon^{\sigma+r_{1}} f_{1}\left(\eta_{e, j}, w_{e, j}\right) \\
& f_{2}\left(\varepsilon^{r_{1}} \eta_{e, j}, \varepsilon^{r_{2}} w_{e, j}\right)=\varepsilon^{\sigma+r_{2}} f_{2}\left(\eta_{e, j}, w_{e, j}\right)
\end{aligned}
$$


with $f_{1}(\cdot)=w_{e, j}, f_{2}=-K_{1} \operatorname{sig}^{\beta_{1}}\left(\eta_{e, j}\right)-K_{2} \operatorname{sig}^{\beta_{2}}\left(w_{e, j}\right)$ and a negative degree of homogeneous with respect to the dilation in (26), i.e., $\sigma=\left(\beta_{1}-1\right) / 2<0$.

By Lemma 1, we can conclude that the tracking error system (14) without disturbances controlled by the HFC scheme (16) is globally finite-time stable, i.e., there exists a finite time $0<T<\infty$ such that

$$
\boldsymbol{\eta}_{e}(t) \equiv 0, \boldsymbol{w}_{e}(t) \equiv 0, \forall t \geq T
$$

Together with (10), we further have

$$
\boldsymbol{\eta}_{e}(t) \equiv 0, \nu_{e}(t) \equiv 0, \forall t \geq T
$$

This concludes the proof.

Remark 2: If the powers $\beta_{1}$ and $\beta_{2}$ of the HFC scheme in (16) are set as $\beta_{1}=\beta_{2}=1$, the closed-loop system composed of the tracking error system (14) without disturbances and the HFC law (16) degrades to be globally asymptotically stable, i.e.,

$$
\begin{aligned}
\dot{\eta}_{e, j} & =w_{e, j} \\
\dot{w}_{e, j} & =-K_{1} \eta_{e, j}-K_{2} w_{e, j}
\end{aligned}
$$

which can be derived easily from the conventional backstepping technique.

Remark 3: Note that the external disturbance $\boldsymbol{d}(t)$ in (14) is not addressed in the nominal HFC scheme. In this context, within the HFC framework, the disturbance observer is expected to be developed for enhancing the robustness and even achieving exact disturbance rejection.

\section{B. Finite-Time Disturbance Observer Based HFC}

In this subsection, the finite-time disturbance observer based HFC (FDO-HFC) scheme is proposed. To this end, a generic assumption on the disturbance $\boldsymbol{d}(t)$ is required as follows:

Assumption 1: The external time-varying disturbance $\boldsymbol{d}(t)$ in (11) satisfies

$$
\boldsymbol{d}^{(n)}(t)=\sum_{i=0}^{n-1} \mathbf{H}_{n-i} \boldsymbol{d}^{(i)}(t), \quad i=0,1, \cdots, n-1
$$

where $n$ is a positive integer and $\mathbf{H}_{i}=\operatorname{diag}\left(h_{i, 1}, h_{i, 2}, h_{i, 3}\right)$ with any constants $h_{i, j} \in \mathbb{R}, j=1,2,3$.

The key result pertaining to the FDO-HFC scheme is now summarized as follows:

Theorem 2 (FDO-HFC): Consider the ASV in (11) with unknown external disturbances $\boldsymbol{d}(t)$ satisfying Assumption 1, an FDO-HFC scheme designed as follows:

$$
\begin{aligned}
\boldsymbol{\tau}_{\mathrm{FDO}}= & -\mathbf{M R}^{-1}\left(K_{1} \operatorname{sig}^{\beta_{1}}\left(\boldsymbol{\eta}-\boldsymbol{\eta}_{d}\right)+K_{2} \operatorname{sig}^{\beta_{2}}\left(\mathbf{R} \boldsymbol{\nu}-\mathbf{R}_{d} \boldsymbol{\nu}_{d}\right)\right) \\
& -\mathbf{M S} \boldsymbol{\nu}+\mathbf{M R}^{-1} \mathbf{S}_{d} \mathbf{R}_{d} \boldsymbol{\nu}_{d} \\
& -\boldsymbol{f}(\boldsymbol{\eta}, \boldsymbol{\nu})+\mathbf{M R}^{-1} \mathbf{R}_{d} \mathbf{M}^{-1} \boldsymbol{f}_{0}\left(\boldsymbol{\eta}_{d}, \boldsymbol{\nu}_{d}\right)-\mathbf{M R} \mathbf{R}^{-1} \widehat{\boldsymbol{d}}
\end{aligned}
$$

with the FDO governed by

$$
\widehat{\boldsymbol{d}}=\widehat{\boldsymbol{p}}_{1}+\mathbf{H}_{1} \widehat{\boldsymbol{p}}_{0}
$$

where $\widehat{\boldsymbol{p}}_{1}$ and $\widehat{\boldsymbol{p}}_{0}$ are derived by

$$
\begin{aligned}
\dot{\widehat{\boldsymbol{p}}}_{0} & =\widehat{\boldsymbol{p}}_{1}+\mathbf{H}_{1} \boldsymbol{p}_{0}+\boldsymbol{u}+\mathbf{L}_{0} \operatorname{sig}^{\alpha_{1}}\left(\boldsymbol{p}_{0}-\widehat{\boldsymbol{p}}_{0}\right) \\
\vdots & \\
\dot{\hat{\boldsymbol{p}}}_{n-1} & =\widehat{\boldsymbol{p}}_{n}+\mathbf{H}_{n} \boldsymbol{p}_{0}-\mathbf{H}_{n-1} \boldsymbol{u}+\mathbf{L}_{n-1} \operatorname{sig}^{\alpha_{n}}\left(\boldsymbol{p}_{0}-\widehat{\boldsymbol{p}}_{0}\right) \\
\dot{\hat{\boldsymbol{p}}}_{n} & =-\mathbf{H}_{n} \boldsymbol{u}+\mathbf{L}_{n} \operatorname{sig}^{\alpha_{n+1}}\left(\boldsymbol{p}_{0}-\widehat{\boldsymbol{p}}_{0}\right)
\end{aligned}
$$

with

$$
\boldsymbol{p}_{0}=\boldsymbol{w}_{e}, \boldsymbol{u}=\mathbf{R M}^{-1} \boldsymbol{\tau}+\boldsymbol{h}_{e}
$$

and $\mathbf{L}_{i}=\operatorname{diag}\left(l_{i, 1}, l_{i, 2}, l_{i, 3}\right), i=0,1, \cdots, n, \alpha_{i}=1+i \vartheta$ with $-1 /(n+1)<\vartheta<0$, and $\vartheta=-q_{1} / q_{2}$ with $q_{1}$ and $q_{2}$ being positive even and odd integers, can render the ASV in (4) exactly track the desired trajectory generated by (9) within a short time $0<T<\infty$, i.e., $\boldsymbol{\eta}(t) \equiv \boldsymbol{\eta}_{d}(t), \boldsymbol{\nu}(t) \equiv \boldsymbol{\nu}_{d}(t), \forall t \geq$ $T$.

Proof: In order to examine finite-time stability of the closed-loop system (14) and (33) including an FDO (35), we need to obtain the disturbance observation error dynamics. To this end, we define auxiliary variables as follows:

$\varepsilon_{0}=\boldsymbol{w}_{e}, \boldsymbol{\varepsilon}_{1}=\boldsymbol{d}(t), \boldsymbol{\varepsilon}_{2}=\dot{\boldsymbol{d}}(t), \cdots, \boldsymbol{\varepsilon}_{n}=\boldsymbol{d}^{(n-1)}(t)$

Together with (14) and (32), we thus have

$$
\begin{aligned}
\dot{\varepsilon}_{0} & =\varepsilon_{1}+\boldsymbol{u} \\
\dot{\varepsilon}_{i} & =\boldsymbol{\varepsilon}_{i+1}, \quad i=1,2, \cdots, n-1 \\
\dot{\boldsymbol{\varepsilon}}_{n} & =\mathbf{H}_{n} \boldsymbol{\varepsilon}_{1}+\mathbf{H}_{n-1} \boldsymbol{\varepsilon}_{2}+\cdots+\mathbf{H}_{1} \boldsymbol{\varepsilon}_{n}
\end{aligned}
$$

Consider a coordinate transformation governed by

$$
\begin{aligned}
\boldsymbol{p}_{0} & =\varepsilon_{0} \\
\boldsymbol{p}_{1} & =\varepsilon_{1}-\mathbf{H}_{1} \varepsilon_{0} \\
& \vdots \\
\boldsymbol{p}_{n} & =\boldsymbol{\varepsilon}_{n}-\mathbf{H}_{1} \varepsilon_{n-1}-\cdots-\mathbf{H}_{n} \varepsilon_{0}
\end{aligned}
$$

we further have

$$
\begin{aligned}
\dot{\boldsymbol{p}}_{0} & =\boldsymbol{p}_{1}+\mathbf{H}_{1} \boldsymbol{p}_{0}+\boldsymbol{u} \\
\vdots & \\
\dot{\boldsymbol{p}}_{n-1} & =\boldsymbol{p}_{n}+\mathbf{H}_{n} \boldsymbol{p}_{0}-\mathbf{H}_{n-1} \boldsymbol{u} \\
\dot{\boldsymbol{p}}_{n} & =-\mathbf{H}_{n} \boldsymbol{u}
\end{aligned}
$$

Together with (35) and (40), the disturbance observation error dynamics can be derived as follows:

$$
\begin{aligned}
\dot{\boldsymbol{p}}_{e, 0} & =\boldsymbol{p}_{e, 1}-\mathbf{L}_{0} \operatorname{sig}^{\alpha_{1}}\left(\boldsymbol{p}_{e, 0}\right) \\
\vdots & \\
\dot{\boldsymbol{p}}_{e, n-1} & =\boldsymbol{p}_{e, n}-\mathbf{L}_{n-1} \operatorname{sig}^{\alpha_{n}}\left(\boldsymbol{p}_{e, 0}\right) \\
\dot{\boldsymbol{p}}_{e, n} & =-\mathbf{L}_{n} \operatorname{sig}^{\alpha_{n+1}}\left(\boldsymbol{p}_{e, 0}\right)
\end{aligned}
$$

where $\boldsymbol{p}_{e, i}=\boldsymbol{p}_{i}-\widehat{\boldsymbol{p}}_{i}:=\left[p_{e, i}^{1}, p_{e, i}^{2}, p_{e, i}^{3}\right]^{T}, i=0,1, \cdots, n$, and

$$
\dot{p}_{e, 0}^{j}=p_{e, 1}^{j}-l_{0, j} \operatorname{sig}^{\alpha_{1}}\left(p_{e, 0}^{j}\right)
$$

$$
\begin{aligned}
\dot{p}_{e, n-1}^{j} & =p_{e, n}^{j}-l_{n-1, j} \operatorname{sig}^{\alpha_{n}}\left(p_{e, 0}^{j}\right) \\
\dot{p}_{e, n}^{j} & =-l_{n, j} \operatorname{sig}^{\alpha_{n+1}}\left(p_{e, 0}^{j}\right), \quad j=1,2,3
\end{aligned}
$$


Applying Lemma 2 to system (42), we can conclude that the disturbance observation errors $\boldsymbol{p}_{e, i}, i=0,1, \cdots, n$ are globally finite-time stable, i.e., the FDO in (35) can exactly observe the dynamics in (40) within a finite time $0<T<\infty$. Together with (34), (37) and (39), we can immediately obtain that $\widehat{\boldsymbol{p}}_{0}$ and $\widehat{\boldsymbol{p}}_{1}$ can exactly estimate $\boldsymbol{w}_{e}$ and $\boldsymbol{d}-\mathbf{H}_{1} \boldsymbol{w}_{e}$, respectively, and $\widehat{d}$ governed by (34) can thus exactly observe the disturbance $d$ in a finite time. Actually, the derivatives $\boldsymbol{d}^{(i)}(t), i=1,2, \cdots, n-1$ can also be exactly observed within a finite time by $\widehat{\boldsymbol{p}}_{i+1}+\mathbf{H}_{1} \widehat{\boldsymbol{p}}_{i}+\cdots+\mathbf{H}_{i+1} \widehat{\boldsymbol{p}}_{0}$.

In this context, we eventually have

$$
\widehat{\boldsymbol{d}}^{(i)}(t) \equiv \boldsymbol{d}^{(i)}(t), \forall t>T, \quad i=0,1, \cdots, n-1
$$

with $\widehat{\boldsymbol{d}}^{(i)}=\widehat{\boldsymbol{p}}_{i+1}+\mathbf{H}_{1} \widehat{\boldsymbol{p}}_{i}+\cdots+\mathbf{H}_{i+1} \widehat{\boldsymbol{p}}_{0}$.

Substituting (33) together with (34) and (43) into (14) yields

$$
\begin{aligned}
\dot{\eta}_{e, j} & =w_{e, j} \\
\dot{w}_{e, j} & =-K_{1} \operatorname{sig}^{\beta_{1}}\left(\eta_{e, j}\right)-K_{2} \operatorname{sig}^{\beta_{2}}\left(w_{e, j}\right)+\tilde{d}_{j}
\end{aligned}
$$

where $\tilde{d}_{j}=d_{j}-\widehat{d}_{j}$.

Together with (43) and (44), we further have

$$
\begin{aligned}
\dot{\eta}_{e, j}(t) & =w_{e, j}(t) \\
\dot{w}_{e, j}(t) & =-K_{1} \operatorname{sig}^{\beta_{1}}\left(\eta_{e, j}(t)\right)-K_{2} \operatorname{sig}^{\beta_{2}}\left(w_{e, j}(t)\right)
\end{aligned}
$$

for any $t>T$ with a finite time $0<T<\infty$.

In what follows, similar to the proof of Theorem 1, global finite-time stability of the closed-loop system (45) can be ensured. As a consequence, in the presence of complex disturbances, using the FDO-HFC in (33), the ASV in (4) can exactly track the desired trajectory $\left(\boldsymbol{\eta}_{d}, \boldsymbol{\nu}_{d}\right)$ generated by (9) in a finite time. This concludes the proof.

Remark 4: In addition to disturbances $\tau_{d}$, the dynamics $\boldsymbol{h}_{e}$ given by (15) might be at least partially unknown due to nonlinearities $f(\cdot)$ and $f_{0}(\cdot)$, and will make the foregoing HFC in (16) and FDO-HFC in (33) unavailable. In this context, the observer for accurate estimate on mixed unknowns including not only unmodeled dynamics but also external disturbances is required to be a patch within the HFC framework.

\section{Finite-Time Unknown Observer Based HFC}

Rewrite the tracking error dynamics in (14) as follows:

$$
\begin{aligned}
\dot{\boldsymbol{\eta}}_{e} & =\boldsymbol{w}_{e} \\
\dot{\boldsymbol{w}}_{e} & =\mathbf{R} \mathbf{M}^{-1} \boldsymbol{\tau}+\mathbf{S} \boldsymbol{w}-\mathbf{S}_{d} \boldsymbol{w}_{d}+\boldsymbol{f}_{u}\left(\boldsymbol{\eta}, \boldsymbol{w}, \boldsymbol{\eta}_{d}, \boldsymbol{w}_{d}, t\right)
\end{aligned}
$$

with the lumped unknowns $f_{u}$ including unmodeled dynamics $f$, desired dynamics $f_{0}$ and disturbances $d$, i.e.,

$$
\begin{aligned}
\boldsymbol{f}_{u}\left(\boldsymbol{\eta}, \boldsymbol{w}, \boldsymbol{\eta}_{d}, \boldsymbol{w}_{d}, t\right)= & \mathbf{R} \mathbf{M}^{-1} \boldsymbol{f}\left(\boldsymbol{\eta}, \mathbf{R}^{T} \boldsymbol{w}\right) \\
& -\mathbf{R}_{d} \mathbf{M}^{-1} \boldsymbol{f}_{0}\left(\boldsymbol{\eta}_{d}, \mathbf{R}_{d}^{T} \boldsymbol{w}_{d}\right)+\boldsymbol{d}(t)
\end{aligned}
$$

From (47), it reasonably requires to assume that dynamics $f, f_{0}$ and $\boldsymbol{d}$ are twice differentiable, and thereby contributing to the following hypothesis:

$$
\left\|\ddot{\boldsymbol{f}}_{u}\right\| \leq L_{u}
$$

for a bounded constant $L_{u}<\infty$.
In this context, a finite-time unknown observer based HFC (FUO-HFC) scheme will be proposed to accurately track the ASV in (4) with complex unknowns including both unmodelded dynamics and disturbances to a desired trajectory with completely unknown dynamics. This challenging problem will be solved by the proposed FUO-HFC scheme with global finite-time stability presented as follows.

Theorem 3 (FUO-HFC): Consider the ASV in (4) with unmodeled dynamics $f$ and unknown external disturbances $\boldsymbol{d}(t)$, an FUO-HFC scheme designed as follows:

$$
\begin{aligned}
\boldsymbol{\tau}_{\mathrm{FUO}}=-\mathbf{M R}^{-1}( & K_{1} \operatorname{sig}^{\beta_{1}}\left(\boldsymbol{\eta}-\boldsymbol{\eta}_{d}\right) \\
& \left.+K_{2} \operatorname{sig}^{\beta_{2}}\left(\mathbf{R} \boldsymbol{\nu}-\mathbf{R}_{d} \boldsymbol{\nu}_{d}\right)+\boldsymbol{z}_{1}\right)
\end{aligned}
$$

with $z_{1}$ estimated by the following FUO:

$$
\begin{aligned}
& \dot{\boldsymbol{z}}_{0}=\boldsymbol{\zeta}_{0}+\mathbf{R M}^{-1} \boldsymbol{\tau}+\mathbf{S} \boldsymbol{w}-\mathbf{S}_{d} \boldsymbol{w}_{d} \\
& \boldsymbol{\zeta}_{0}=-\lambda_{1} \mathcal{L}^{1 / 3} \operatorname{sig}^{2 / 3}\left(\boldsymbol{z}_{0}-\boldsymbol{w}_{e}\right)+\boldsymbol{z}_{1} \\
& \dot{\boldsymbol{z}}_{1}=\boldsymbol{\zeta}_{1} \\
& \boldsymbol{\zeta}_{1}=-\lambda_{2} \mathcal{L}^{1 / 2} \operatorname{sig}^{1 / 2}\left(\boldsymbol{z}_{1}-\boldsymbol{\zeta}_{0}\right)+\boldsymbol{z}_{2} \\
& \dot{\boldsymbol{z}}_{2}=-\lambda_{3} \mathcal{L} \operatorname{sgn}\left(\boldsymbol{z}_{2}-\boldsymbol{\zeta}_{1}\right)
\end{aligned}
$$

where $z_{j}:=\left[z_{j, 1}, z_{j, 2}, z_{j, 3}\right]^{T}, j=0,1,2, \quad \zeta_{k}:=$ $\left[\zeta_{k, 1}, \zeta_{k, 2}, \zeta_{k, 3}\right]^{T}, k=0,1, \lambda_{i}>0, i=1,2,3$ and $\mathcal{L}=$ $\operatorname{diag}\left(\ell_{1}, \ell_{2}, \ell_{3}\right)$, can render the ASV exactly track the desired trajectory generated by (9) with completely unknown dynamics $\boldsymbol{f}_{0}$ in a short time $0<T<\infty$, i.e., $\boldsymbol{\eta}(t) \equiv \boldsymbol{\eta}_{d}(t), \boldsymbol{\nu}(t) \equiv$ $\boldsymbol{\nu}_{d}(t), \forall t \geq T$.

Proof: Define the FUO observation errors as follows:

$$
e_{1}=z_{0}-w_{e}, e_{2}=z_{1}-f_{u}, e_{3}=z_{2}-\dot{f}_{u}
$$

Combining with (46) and (50), we have the FUO observation error dynamics as follows:

$$
\begin{aligned}
& \dot{\boldsymbol{e}}_{1}=-\lambda_{1} \mathcal{L}^{1 / 3} \operatorname{sig}^{2 / 3}\left(\boldsymbol{e}_{1}\right)+\boldsymbol{e}_{2} \\
& \dot{\boldsymbol{e}}_{2}=-\lambda_{2} \mathcal{L}^{1 / 2} \operatorname{sig}^{1 / 2}\left(\boldsymbol{e}_{2}-\dot{\boldsymbol{e}}_{1}\right)+\boldsymbol{e}_{3} \\
& \dot{\boldsymbol{e}}_{3}=-\lambda_{3} \mathcal{L} \operatorname{sgn}\left(\boldsymbol{e}_{3}-\dot{\boldsymbol{e}}_{2}\right)-\ddot{\boldsymbol{f}}_{u}
\end{aligned}
$$

i.e.,

$$
\begin{aligned}
& \dot{e}_{1, j}=-\lambda_{1} \ell_{j}^{1 / 3} \operatorname{sig}^{2 / 3}\left(e_{1, j}\right)+e_{2, j} \\
& \dot{e}_{2, j}=-\lambda_{2} \ell_{j}^{1 / 2} \operatorname{sig}^{1 / 2}\left(e_{2, j}-\dot{e}_{1, j}\right)+e_{3, j} \\
& \dot{e}_{3, j} \in-\lambda_{3} \ell_{j} \operatorname{sgn}\left(e_{3, j}-\dot{e}_{2, j}\right)+\left[-L_{u}, L_{u}\right]
\end{aligned}
$$

where " $\in$ " denotes the differential inclusion understood in the Filippov sense [41].

According to [42, Lemma 2] together with its detailed proof, we can immediately conclude that the tracking error dynamics in (53) is globally finite-time stable, i.e., there exists a finite time $0<T<\infty$ such that

$$
\boldsymbol{z}_{0}(t) \equiv \boldsymbol{w}_{e}(t), \boldsymbol{z}_{1}(t) \equiv \boldsymbol{f}_{u}(t), \boldsymbol{z}_{2}(t) \equiv \dot{\boldsymbol{f}}_{u}(t), \forall t>T
$$

Substituting (49) into (46) and using (54) yields

$$
\begin{aligned}
\dot{\boldsymbol{\eta}}_{e}(t) & =\boldsymbol{w}_{e}(t) \\
\dot{\boldsymbol{w}}_{e}(t) & =-K_{1} \operatorname{sig}^{\beta_{1}}\left(\boldsymbol{\eta}_{e}(t)\right)-K_{2} \operatorname{sig}^{\beta_{2}}\left(\boldsymbol{w}_{e}(t)\right), \forall t>T
\end{aligned}
$$


TABLE I: Main parameters of CyberShip II

\begin{tabular}{lrlrlr}
\hline$m$ & 23.8000 & $Y_{v}$ & -0.8612 & $X_{\dot{u}}$ & -2.0 \\
$I_{z}$ & 1.7600 & $Y_{|v| v}$ & -36.2823 & $Y_{\dot{v}}$ & -10.0 \\
$x_{g}$ & 0.0460 & $Y_{r}$ & 0.1079 & $Y_{\dot{r}}$ & -0.0 \\
$X_{u}$ & -0.7225 & $N_{v}$ & 0.1052 & $N_{\dot{v}}$ & -0.0 \\
$X_{|u| u}$ & -1.3274 & $N_{|v| v}$ & 5.0437 & $N_{\dot{r}}$ & -1.0 \\
$X_{u u u}$ & -5.8664 & & & & \\
\hline
\end{tabular}

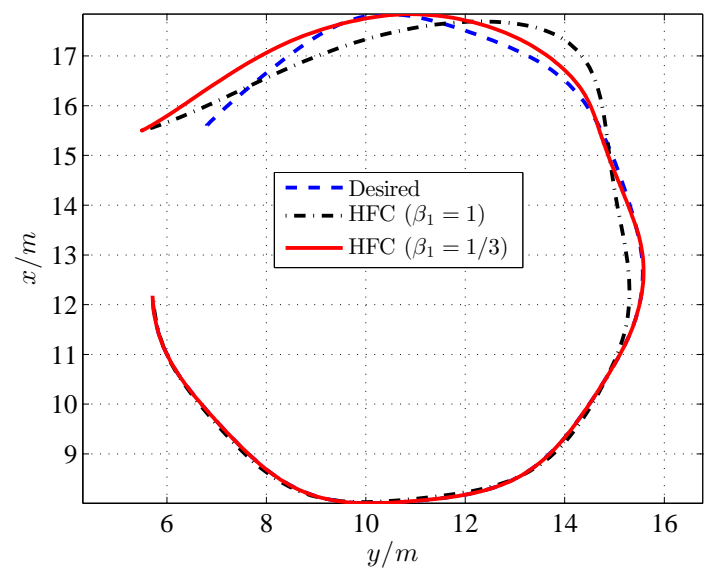

Fig. 2: Desired and actual trajectories in the $x y$ plane

which has been proven to be globally finite-time stable in Theorem 1. In this context, the proposed FUO-HFC in (49) renders the ASV in (4) with lumped unmodeled dynamics and disturbances can exactly track the desired trajectory $\left(\boldsymbol{\eta}_{d}, \boldsymbol{\nu}_{d}\right)$ generated by (9) with completely unknown dynamics in a finite time. This concludes the proof.

Remark 5: It should be noted that in addition to unmodeled dynamics and external disturbances pertaining to the ASV, the desired dynamics of the trajectory to be tracked are also not necessarily known for the FUO-HFC scheme. It implies that the proposed FUO-HFC approach is completely independent on dynamics of the ASV and the desired trajectory, and thereby contributing to a both task-free and model-free methodology.

\section{Simulation Studies And Discussions}

In order to demonstrate the effectiveness and superiority of the proposed control schemes for trajectory tracking control of an ASV, simulation studies and comprehensive comparisons are conducted on a well-known surface vehicle CyberShip II [43] of which the main parameters are listed in Table I.

Our objective is to track exactly the desired trajectory $\left(\boldsymbol{\eta}_{d}, \boldsymbol{\nu}_{d}\right)$ governed by (9) with assumed dynamics $\boldsymbol{f}_{0}\left(\boldsymbol{\eta}_{d}, \boldsymbol{\nu}_{d}\right)=$ $-\mathbf{C}\left(\boldsymbol{\nu}_{d}\right) \boldsymbol{\nu}_{d}-\mathbf{D}\left(\boldsymbol{\nu}_{d}\right) \boldsymbol{\nu}_{d}-\mathbf{g}\left(\boldsymbol{\eta}_{d}, \boldsymbol{\nu}_{d}\right)+\boldsymbol{\tau}_{0}$, where $\boldsymbol{\tau}_{0}=$ $\left[6,3 \cos ^{2}(0.2 \pi t), \sin ^{2}(0.2 \pi t)\right]^{T}$, and the initial conditions are set as $\boldsymbol{\eta}_{d}(0)=[15.6,6.8, \pi / 4]^{T}, \boldsymbol{\nu}_{d}(0)=[1,0,0]^{T}, \boldsymbol{\eta}(0)=$ $[15.5,5.5, \pi / 2]^{T}$ and $\boldsymbol{\nu}(0)=[0,0,0]^{T}$.

In what follows, 3 cases will be deployed to evaluate the performance of the proposed HFC, FDO-HFC and FUO-HFC approaches, respectively.
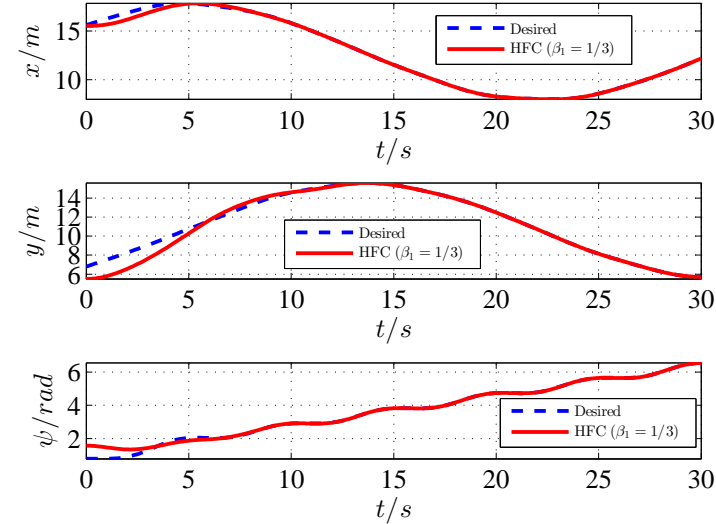

Fig. 3: Desired and actual states $x, y$, and $\psi$
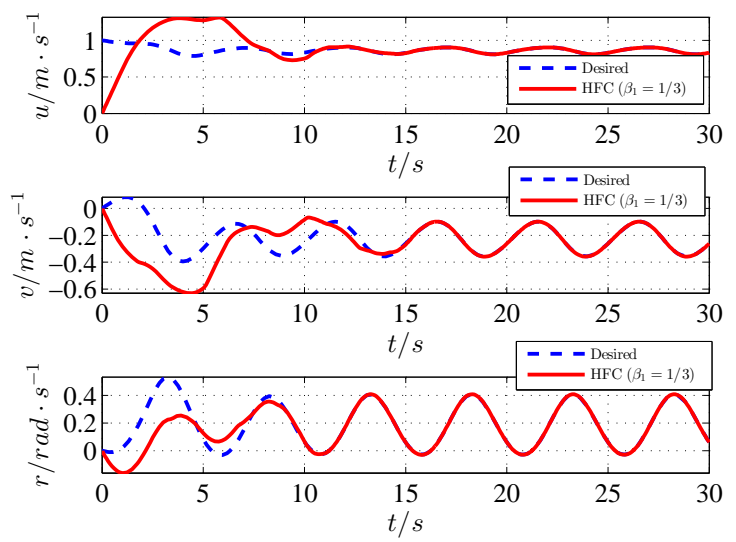

Fig. 4: Desired and actual states $u, v$, and $r$
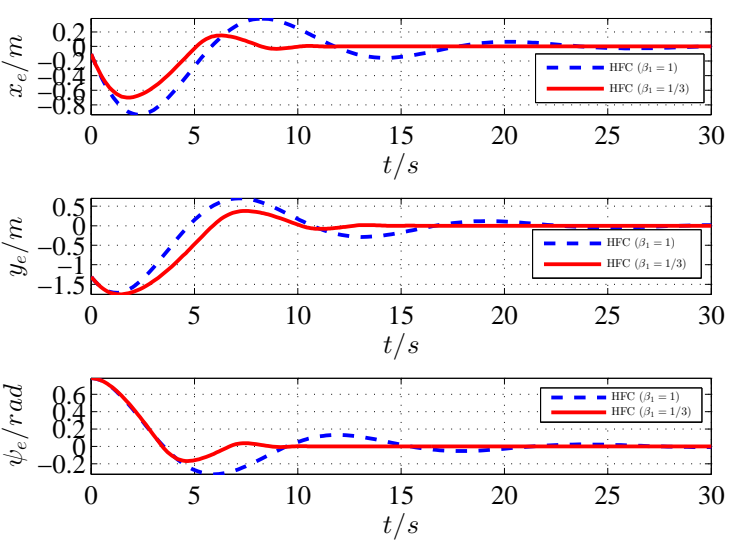

Fig. 5: Tracking errors $x_{e}, y_{e}$, and $\psi_{e}$

\section{A. Performance Evaluation on the HFC}

In this subsection, a nominal case where the ASV is sufficiently modeled (i.e., $f$ is known) and external disturbances are not considered (i.e., $\boldsymbol{d}=0$ ) is employed to demonstrate the effectiveness and superiority of the proposed HFC scheme. 

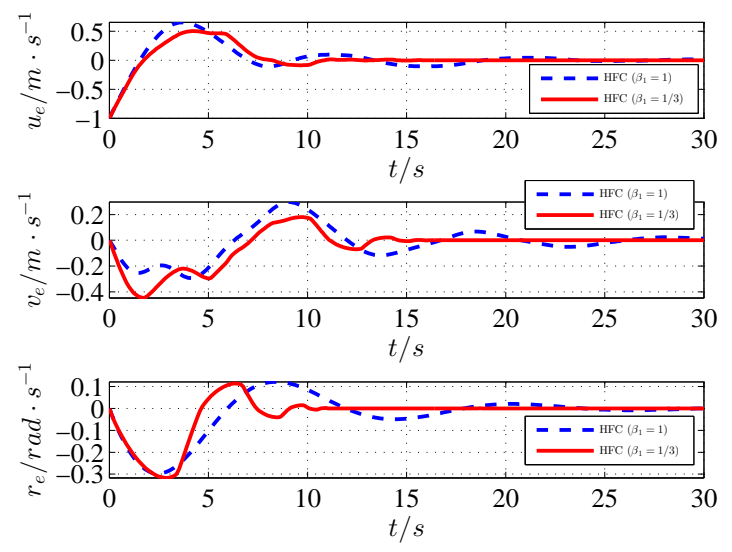

Fig. 6: Tracking errors $u_{e}, v_{e}$, and $r_{e}$
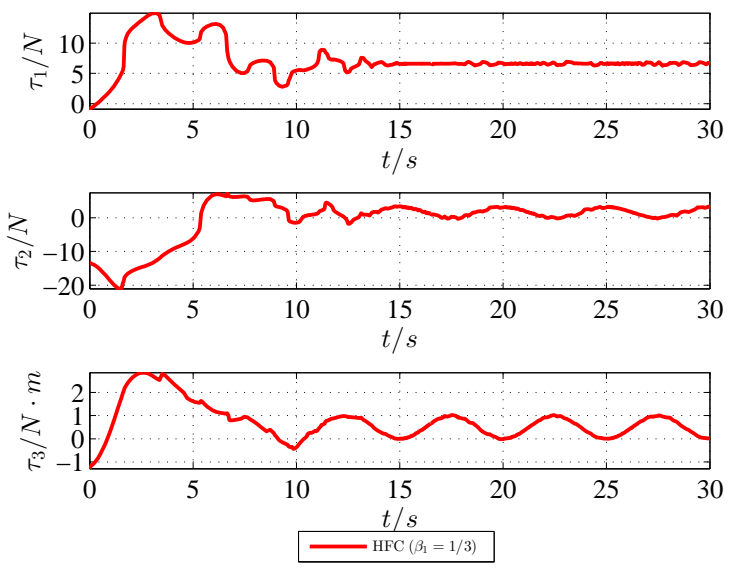

Fig. 7: Control forces $\tau_{1}, \tau_{2}$, and torque $\tau_{3}$

User-defined parameters are chosen as follows: $K_{1}=0.3$, $K_{2}=0.3, \beta_{1}=1 / 3$ and $\beta_{2}=1 / 2$.

Simulation results are shown in Figs. 2-7. Comparing with the traditional asymptotic approach, i.e., $\beta_{1}=\beta_{2}=1$ within the HFC scheme, we can see from Fig. 2 that the HFC with $\beta_{1}=1 / 3$ can achieve much faster convergence. In addition, as shown in Figs. 3-6, the ASV can exactly track the desired trajectory within a finite time by virtue of the HFC laws shown in Fig. 7. In comparison with the asymptotic approach (i.e., $\beta_{1}=1$ ), the HFC scheme with $\beta_{1}=1 / 3$ is able to render tracking errors converge to the origin in a very short time.

\section{B. Performance Evaluation on the FDO-HFC}

In this subsection, a much more practical case with unknown disturbances is deployed to demonstrate the performance evaluation and comparisons. In order to facilitate simulation studies, the external disturbances are assumed to be governed by

$$
\boldsymbol{d}(t)=\left[\begin{array}{l}
10 \cos (0.1 \pi t-\pi / 5) \\
8 \cos (0.3 \pi t+\pi / 6) \\
6 \cos (0.2 \pi t+\pi / 3)
\end{array}\right]
$$

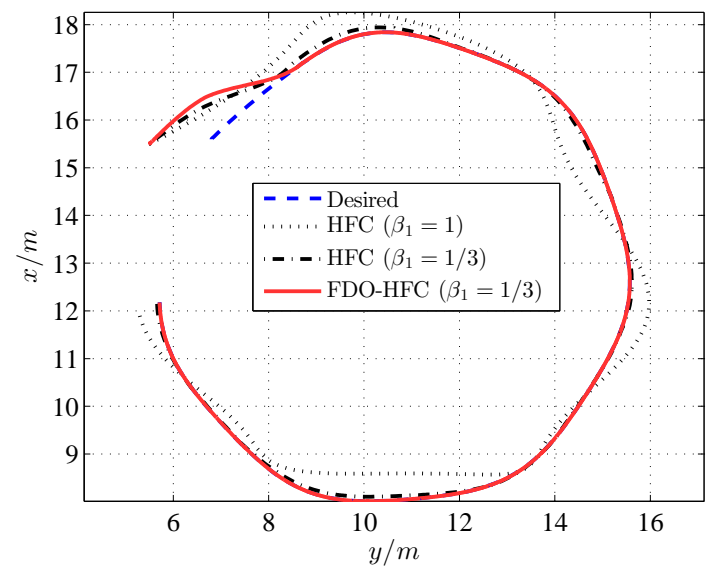

Fig. 8: Desired and actual trajectories in the $x y$ plane
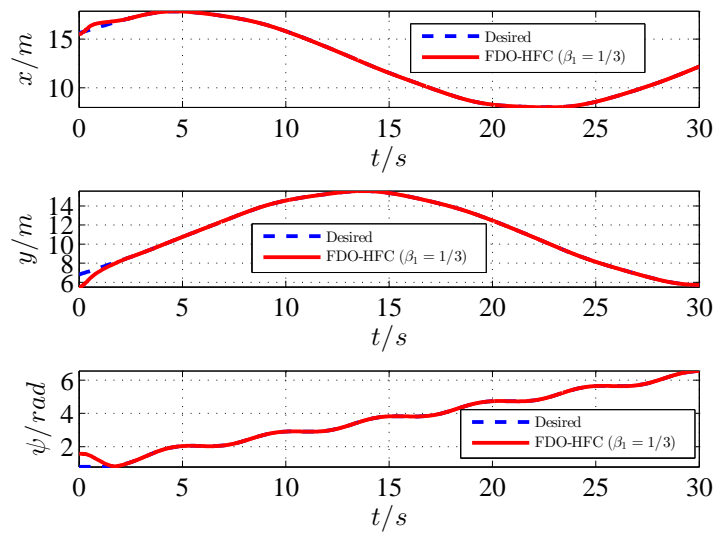

Fig. 9: Desired and actual states $x, y$, and $\psi$
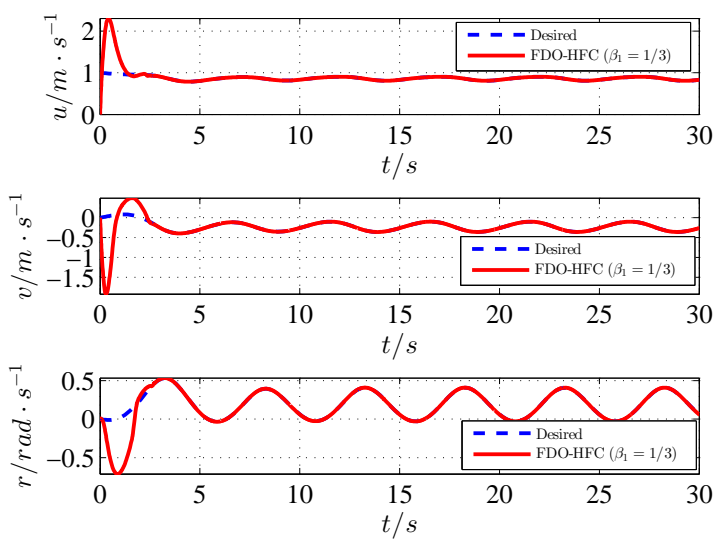

Fig. 10: Desired and actual states $u, v$, and $r$

with $\mathbf{H}_{1}=\operatorname{diag}(0,0,0)$ and $\mathbf{H}_{2}=$ $\operatorname{diag}\left(-0.01 \pi^{2},-0.09 \pi^{2},-0.04 \pi^{2}\right)$.

Accordingly, user-defined parameters of the HFC and FDOHFC schemes are commonly chosen as $K_{1}=2.6, K_{2}=$ $2.6, \beta_{1}=1 / 3$ and $\beta_{2}=1 / 2$. The other parameters of the FDO are selected as follows: $\mathbf{L}_{0}=\operatorname{diag}(10,10,10), \mathbf{L}_{1}=$ 

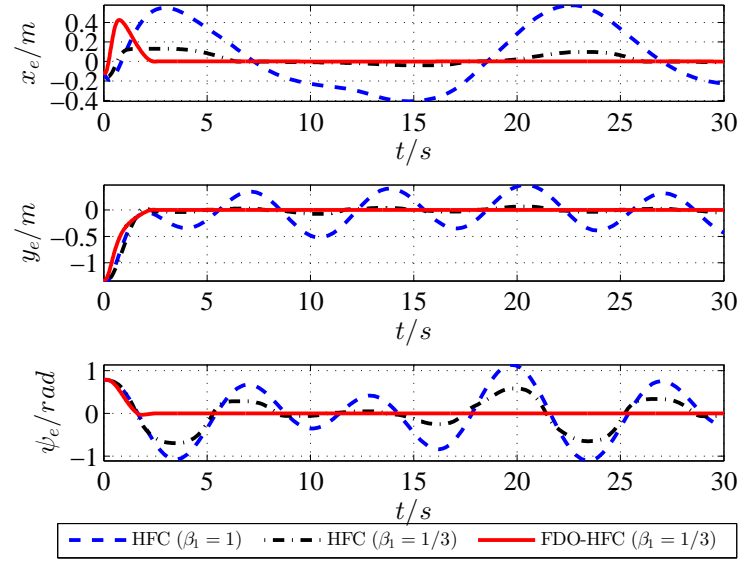

Fig. 11: Tracking errors $x_{e}, y_{e}$, and $\psi_{e}$
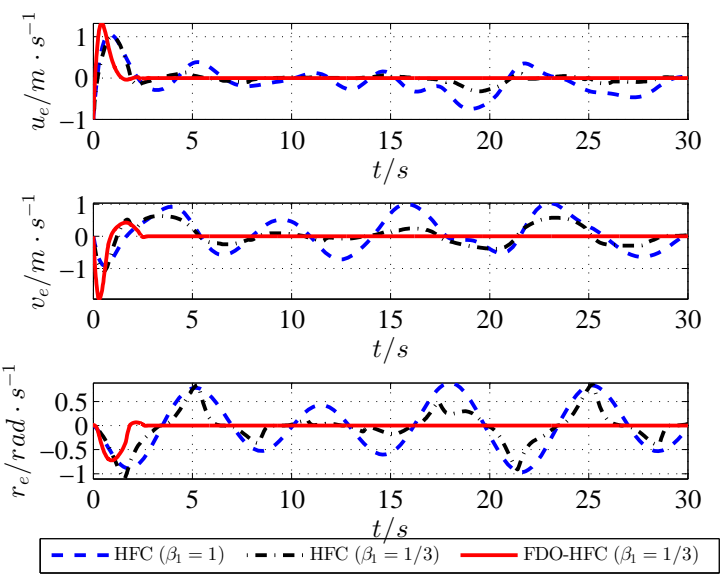

Fig. 12: Tracking errors $u_{e}, v_{e}$, and $r_{e}$
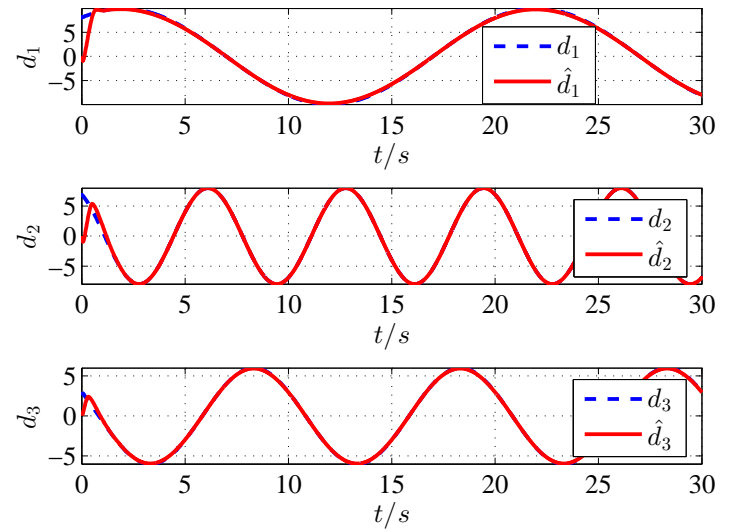

Fig. 13: Disturbances and their finite-time observation

$\operatorname{diag}(32,32,32), \mathbf{L}_{2}=\operatorname{diag}(20,20,20), \alpha_{1}=7 / 9, \alpha_{2}=5 / 9$ and $\alpha_{3}=1 / 3$.

The actual and desired trajectories in the planar space are shown in Fig. 8-12, from which we can see that, in comparison with the conventional asymptotic control scheme (i.e., $\beta_{1}=1$ ), the proposed HFC scheme (i.e., $\beta_{1}=1 / 3$ ) achieves faster

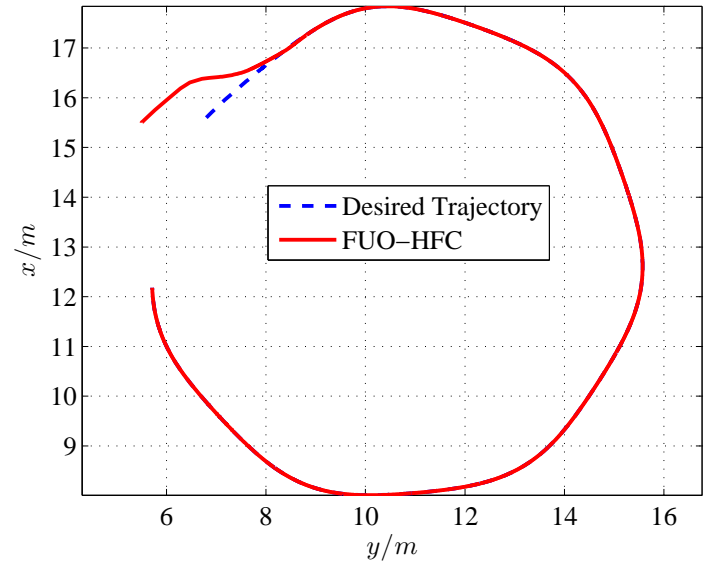

Fig. 14: Desired and actual trajectories in the $x y$ plane
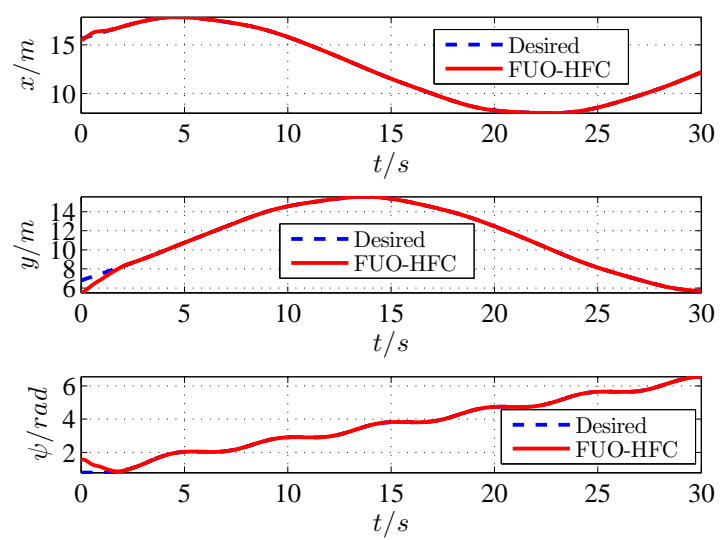

Fig. 15: Desired and actual states $x, y$, and $\psi$
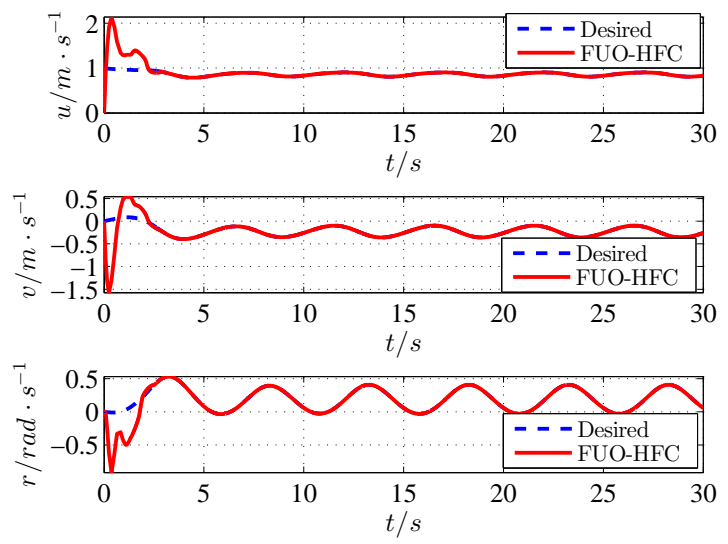

Fig. 16: Desired and actual states $u, v$, and $r$

convergence and stronger disturbance rejection simultaneously, and thereby resulting in higher tracking accuracy, and the FDO-HFC approach is able to realize exact trajectory tracking within a short time since unknown disturbances can be finitetime observed exactly as shown in Fig. 13. 

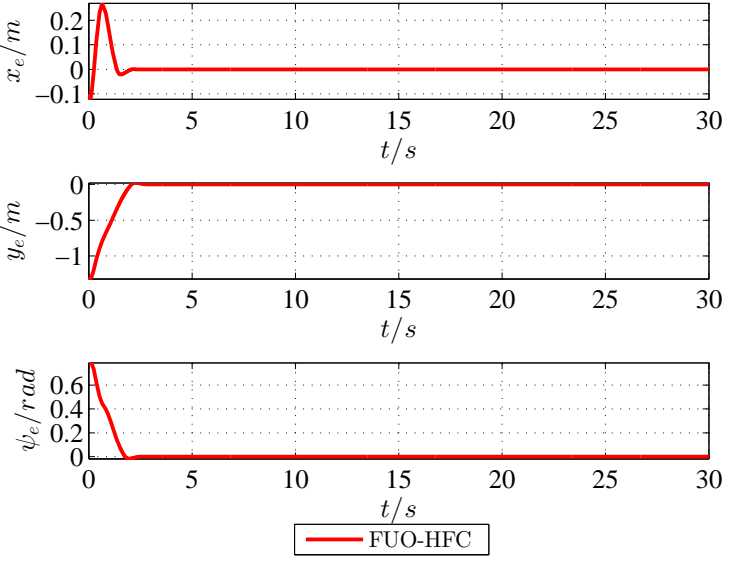

Fig. 17: Tracking errors $x_{e}, y_{e}$, and $\psi_{e}$
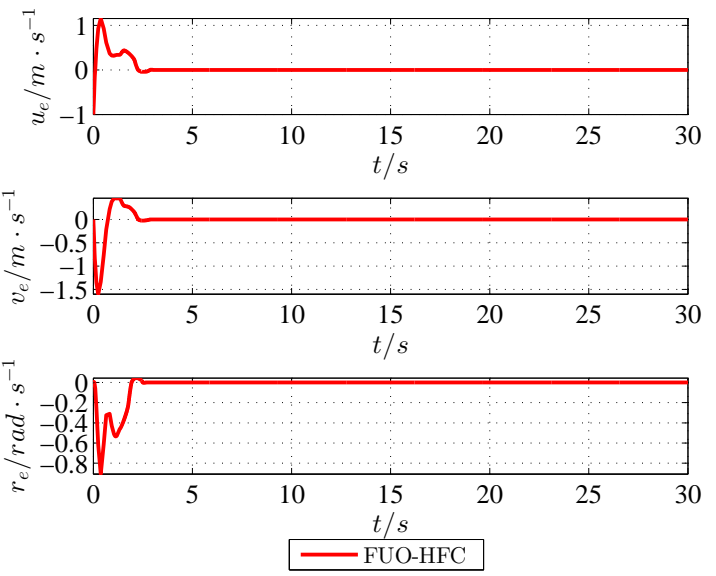

Fig. 18: Tracking errors $u_{e}, v_{e}$, and $r_{e}$
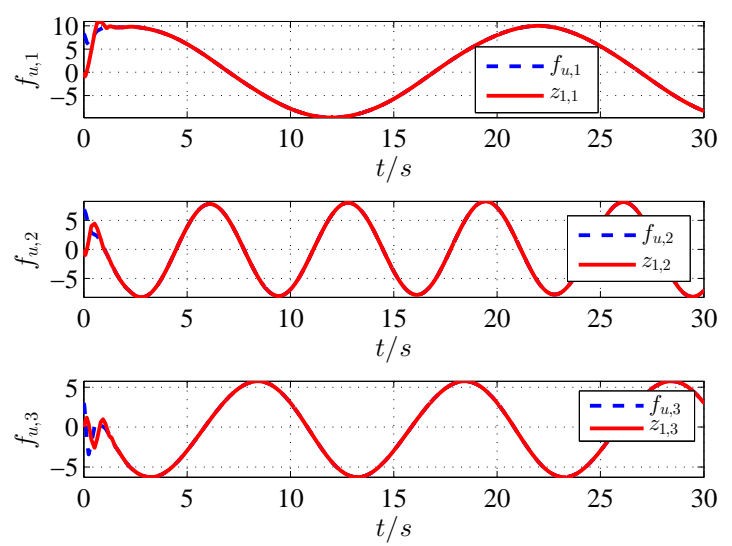

Fig. 19: Lumped unknowns and their finite-time observation

\section{Performance Evaluation on the FUO-HFC}

In order to demonstrate the superiority of the proposed FUO-HFC scheme, we employ a much more complex case where both unmodeled dynamics of the ASV and desired trajectory and unknown disturbances are included. The control parameters are selected as follows: $K_{1}=2.6, K_{2}=2.6$, $\beta_{1}=1 / 3, \beta_{2}=1 / 2, \lambda_{1}=2, \lambda_{2}=1.5, \lambda_{3}=1.1$ and $\mathcal{L}=\operatorname{diag}(30,30,30)$. Corresponding simulation results and comparisons are shown in Figs. 14-19. The actual and reference trajectories in the planar space are shown in Fig. 14, which indicates that the actual trajectory can exactly track the desired one in a very short time although the ASV suffers from unmodeled dynamics and unknown disturbances. Actually, the previous HFC and FDO-HFC approaches become unavailable due to the unexpected unmodeled dynamics of the ASV and the desired trajectory. From the tracking performance on position and velocity shown in Figs. 15-18, we can see that trajectory tracking errors converge to zero in a very short time in spite of complex unknowns including unmodeled dynamics and unknown disturbances. In essence, the remarkable performance of the proposed FUO-HFC scheme on exact trajectory tracking relies on the accurate observation on the lumped unknowns via a FUO, whereby the finite-time observation results are shown in Fig. 19. In this context, the FUO-HFC methodology can achieve fast and exact trajectory tracking together with accurate reconstruction on complex unknowns including unmodeled dynamics, uncertainties and unknown disturbances.

\section{Conclusions}

In this paper, in order to achieve trajectory fast and accurate tracking control of an autonomous surface vehicle (ASV) subject to unmodeled dynamics and unknown disturbances, a homogeneity-based finite-time control (HFC) framework has been innovatively proposed. For exactly dealing with external disturbances, a finite-time disturbance observer (FDO) has been developed and has been incorporated into the HFC framework, thereby contributing the FDO-based HFC (termed FDO-HFC) scheme which can realize exact trajectory tracking control of an ASV in the presence of complex disturbances. To further accurately handle complicated unknowns including both unmodeled dynamics and unknown disturbances, a finitetime unknown observer based HFC (FUO-HFC) scheme has been proposed to enhance the entire performance including both trajectory tracking and unknowns identification, whereby high accuracy and fast convergence can be ensured simultaneously. Simulation studies and comprehensive comparisons have been conducted on a benchmark ship, i.e., CyberShip II, and have demonstrated the effectiveness and superiority of the proposed HFC schemes in term of exact trajectory tracking and unknowns rejection.

\section{ACKNOWLEDGMENTS}

The authors would like to thank the Editor-in-Chief, Associate Editor and anonymous referees for their invaluable comments and suggestions.

\section{APPENDIX A \\ PROOF OF LEMMA 2}

Proof: In the light of Lemma 1, the entire proof can be divided into 2 phases, i.e., proves of global asymptotic stability and negative homogeneity. 


\section{Phase I: Global Asymptotic Stability}

In order to facilitate an inductive proof, applying a set of coordinate transformations as follows:

$$
z_{i}=l_{i-1} x_{i}, \quad i=1,2, \cdots, n
$$

with $l_{0}=1$, to system (2), we have

$$
\begin{aligned}
& \dot{x}_{1}=c_{1}\left(x_{2}-x_{1}^{\alpha_{2}}\right) \\
& \dot{x}_{2}=c_{2}\left(x_{3}-x_{1}^{\alpha_{3}}\right) \\
& \quad \vdots \\
& \dot{x}_{n}=-c_{n} x_{1}^{\alpha_{n+1}}
\end{aligned}
$$

where $x_{1}^{\alpha_{i}}:=\operatorname{sig}^{\alpha_{i}}\left(x_{1}\right)$ and $c_{i}=l_{i} / l_{i-1}, i=1,2, \cdots, n$.

Using (A.2), a backward recursive procedure will be established in the sequel.

Initial step: We first consider the following system:

$$
\dot{x}_{n}=-c_{n} x_{n}^{\alpha_{n+1} / \alpha_{n}}
$$

Choosing a Lyapunov function as follows:

$$
V_{n}\left(x_{n}\right)=\frac{\alpha_{n}}{2}\left|x_{n}\right|^{2 / \alpha_{n}}
$$

we have

$$
\left.\dot{V}_{n}\left(x_{n}\right)\right|_{(A .3)} \leq-k_{n}\left|x_{n}\right|^{(2+\tau) / \alpha_{n}}
$$

with $k_{n}=c_{n}$.

Inductive step: Assume there exists a Lyapunov function as follows:

$$
\begin{aligned}
& V_{i+1}\left(x_{i+1}, x_{i+2}, \cdots, x_{n}\right) \\
& \quad=\sum_{j=i+1}^{n} \int_{x_{j+1}^{\alpha_{j} / \alpha_{j+1}}}^{x_{j}}\left(s^{\left(2-\alpha_{j}\right) / \alpha_{j}}-x_{j+1}^{\left(2-\alpha_{j}\right) / \alpha_{j+1}}\right) d s
\end{aligned}
$$

such that $\dot{V}_{i+1}$ along the following system:

$$
\begin{gathered}
\dot{x}_{i+1}=c_{i+1}\left(x_{i+2}-x_{i+1}^{\alpha_{i+2} / \alpha_{i+1}}\right) \\
\dot{x}_{i+2}=c_{i+2}\left(x_{i+3}-x_{i+1}^{\alpha_{i+3} / \alpha_{i+1}}\right) \\
\vdots \\
\dot{x}_{n}=-c_{n} x_{i+1}^{\alpha_{n+1} / \alpha_{i+1}}
\end{gathered}
$$

satisfies

$$
\left.\dot{V}_{i+1}\right|_{(A .7)} \leq-k_{i+1} \sum_{j=i+1}^{n}\left|x_{j}^{\alpha_{j+1} / \alpha_{j}}-x_{j+1}\right|^{(2+\tau) / \alpha_{j+1}}
$$

with $k_{i+1}>0$. It is easily verified that (A.8) holds for system (A.3) at the initial step, i.e., $i=n-1$.

In this context, we are expected to prove that for the system as follows:

$$
\begin{aligned}
\dot{x}_{i} & =c_{i}\left(x_{i+1}-x_{i}^{\alpha_{i+1} / \alpha_{i}}\right) \\
\dot{x}_{i+1} & =c_{i+1}\left(x_{i+2}-x_{i}^{\alpha_{i+2} / \alpha_{i}}\right) \\
\vdots & \\
\dot{x}_{n} & =-c_{n} x_{i}^{\alpha_{n+1} / \alpha_{i}}
\end{aligned}
$$

there exists the following Lyapunov function:

$$
\begin{gathered}
V_{i}\left(x_{i}, x_{i+1}, \cdots, x_{n}\right)=V_{i+1}\left(x_{i+1}, x_{i+2}, \cdots, x_{n}\right) \\
+\int_{x_{i+1}^{\alpha_{i} / \alpha_{i+1}}}^{x_{i}}\left(s^{\left(2-\alpha_{i}\right) / \alpha_{i}}-x_{i+1}^{\left(2-\alpha_{i}\right) / \alpha_{i+1}}\right) d s
\end{gathered}
$$

such that $\dot{V}_{i}$ along (A.9) satisfies the form like (A.8).

To this end, together with (A.8), we have

$$
\begin{gathered}
\left.\dot{V}_{i}\right|_{(A .9)}=\left.\dot{V}_{i+1}\right|_{(A .7)}-\sum_{j=1+1}^{n} c_{j} \frac{\partial V_{i+1}}{\partial x_{j}}\left(x_{i}^{\alpha_{j+1} / \alpha_{i}}-x_{i+1}^{\alpha_{j+1} / \alpha_{i+1}}\right) \\
+\left.\frac{d}{d t} \int_{x_{i+1}^{\alpha_{i} / \alpha_{i+1}}}^{x_{i}}\left(s^{\left(2-\alpha_{i}\right) / \alpha_{i}}-x_{i+1}^{\left(2-\alpha_{i}\right) / \alpha_{i+1}}\right) d s\right|_{(A .9)} \\
\leq-k_{i+1} \sum_{j=i+1}^{n}\left|x_{j}^{\alpha_{j+1} / \alpha_{j}}-x_{j+1}\right|^{(2+\tau) / \alpha_{j+1}} \\
-c_{i}\left(x_{i}^{\left(2-\alpha_{i}\right) / \alpha_{i}}-x_{i+1}^{\left(2-\alpha_{i}\right) / \alpha_{i+1}}\right)\left(x_{i}^{\alpha_{i+1} / \alpha_{i}}-x_{i+1}\right) \\
-\frac{c_{i+1}\left(2-\alpha_{i}\right)}{\alpha_{i+1}} x_{i+1}^{\left(2-\alpha_{i}-\alpha_{i+1}\right) / \alpha_{i+1}} \\
\quad \times\left(x_{i+2}-x_{i}^{\alpha_{i+2} / \alpha_{i}}\right)\left(x_{i}-x_{i+1}^{\alpha_{i} / \alpha_{i+1}}\right) \\
-\sum_{j=1+1}^{n} c_{j} \frac{\partial V_{i+1}}{\partial x_{j}}\left(x_{i}^{\alpha_{j+1} / \alpha_{i}}-x_{i+1}^{\alpha_{j+1} / \alpha_{i+1}}\right)
\end{gathered}
$$

Note that

$$
\begin{aligned}
& -c_{i}\left(x_{i}^{\left(2-\alpha_{i}\right) / \alpha_{i}}-x_{i+1}^{\left(2-\alpha_{i}\right) / \alpha_{i+1}}\right)\left(x_{i}^{\alpha_{i+1} / \alpha_{i}}-x_{i+1}\right) \\
\leq & -c_{i} k_{i+1}\left|x_{i}^{\alpha_{i+1} / \alpha_{i}}-x_{i+1}\right|^{(2+\tau) / \alpha_{i+1}} \\
& -\frac{c_{i+1}\left(2-\alpha_{i}\right)}{\alpha_{i+1}} x_{i+1}^{\left(2-\alpha_{i}-\alpha_{i+1}\right) / \alpha_{i+1}} \\
& \times\left(x_{i+2}-x_{i}^{\alpha_{i+2} / \alpha_{i}}\right)\left(x_{i}-x_{i+1}^{\left.\alpha_{i} / \alpha_{i+1}\right)}\right. \\
\leq & \mu_{1} \sum_{j=i+1}^{n}\left|x_{j}^{\alpha_{j+1} / \alpha_{j}}-x_{j+1}\right|^{(2+\tau) / \alpha_{j+1}} \\
& +\rho_{1}\left(\mu_{1}\right)\left|x_{i}^{\alpha_{i+1} / \alpha_{i}}-x_{i+1}\right|^{(2+\tau) / \alpha_{i+1}} \\
& -\sum_{j=1+1}^{n} c_{j} \frac{\partial V_{i+1}}{\partial x_{j}}\left(x_{i}^{\alpha_{j+1} / \alpha_{i}}-x_{i+1}^{\alpha_{j+1} / \alpha_{i+1}}\right) \\
\leq & \mu_{2} \sum_{j=i+1}^{n}\left|x_{j}^{\alpha_{j+1} / \alpha_{j}}-x_{j+1}\right|^{(2+\tau) / \alpha_{j+1}} \\
& +\rho_{2}\left(\mu_{2}\right)\left|x_{i}^{\alpha_{i+1} / \alpha_{i}}-x_{i+1}\right|^{(2+\tau) / \alpha_{i+1}}
\end{aligned}
$$

with positive continuous functions $\rho_{1}(\cdot)$ and $\rho_{2}(\cdot)$ with respect to any positive constants $\mu_{1}$ and $\mu_{2}$, respectively.

Substituting (A.12)-(A.14) into (A.11) yields

$$
\begin{aligned}
\left.\dot{V}_{i}\right|_{(A .9)} \leq & -\left(k_{i+1}-\mu_{1}-\mu_{2}\right) \sum_{j=i+1}^{n}\left|x_{j}^{\alpha_{j+1} / \alpha_{j}}-x_{j+1}\right|^{(2+\tau) / \alpha_{j+1}} \\
& -\left(c_{i} k_{i+1}-\rho_{1}-\rho_{2}\right)\left|x_{i}^{\alpha_{i+1} / \alpha_{i}}-x_{i+1}\right|^{(2+\tau) / \alpha_{i+1}}
\end{aligned}
$$

Selecting parameters $\mu_{1}, \mu_{2}, k_{i+1}$ and $c_{i}$ such that

$c_{i} \geq \frac{k_{i}+\rho_{1}\left(\mu_{1}\right)+\rho_{2}\left(\mu_{2}\right)}{k_{i+1}}, k_{i} \leq k_{i+1}-\mu_{1}-\mu_{2}$ 
yields

$$
\left.\dot{V}_{i}\right|_{(A .9)} \leq-k_{i} \sum_{j=i}^{n}\left|x_{j}^{\alpha_{j+1} / \alpha_{j}}-x_{j+1}\right|^{(2+\tau) / \alpha_{j+1}}
$$

which implies that (A.8) also holds for the $i$ th inductive step.

Recursively, we can eventually construct a Lyapunov function as follows:

$$
\begin{aligned}
& V_{1}\left(x_{1}, x_{2}, \cdots, x_{n}\right) \\
& \quad=\sum_{j=1}^{n} \int_{x_{j+1}^{\alpha_{j} / \alpha_{j+1}}}^{x_{j}}\left(s^{\left(2-\alpha_{j}\right) / \alpha_{j}}-x_{j+1}^{\left(2-\alpha_{j}\right) / \alpha_{j+1}}\right) d s
\end{aligned}
$$

with $x_{n+1}=0$ and $\alpha_{1}=1$, such that

$$
\left.\dot{V}_{1}\right|_{(A .2)} \leq-k_{1} \sum_{j=1}^{n}\left|x_{j}^{\alpha_{j+1} / \alpha_{j}}-x_{j+1}\right|^{(2+\tau) / \alpha_{j+1}}
$$

with $k_{1}>0$ recursively determined by (A.16).

It follows from (A.19) that system (A.2) is globally asymptotically stable. Together with the global diffeomorphism (A.1), we have system (2) is globally asymptotically stable.

\section{Phase II: Negative Homogeneity}

Applying a dilation as follows:

$$
\left(r_{1}, r_{2}, \cdots, r_{n}\right)=\left(\alpha_{1}, \alpha_{2}, \cdots, \alpha_{n}\right)
$$

with $\alpha_{1}=1$, to system (2) yields a negative homogeneous of degree, i.e., $\tau=\alpha_{i+1}-\alpha_{i}<0, i=1,2, \cdots, n$.

In this context, combining with Phases I and II, and using Lemma 1, we immediately have system (2) is globally asymptotically stable. This concludes the proof.

\section{REFERENCES}

[1] L. Moreira, T. I. Fossen, and C. G. Soares, "Path following control system for a tanker ship model," Ocean Engineering, vol. 34, no. 14, pp. 2074 2085, Oct. 2007.

[2] J. C. Sun, N. Wang, and M. J. Er, "Extreme learning control of surface vehicles with unknown dynamics and disturbances," Neurocomputing, vol. 167, pp. 535-542, Nov. 2015.

[3] T. I. Fossen, Marine Control Systems: Guidence, Navigation and Control of Ships, Rigs and Underwater Vehicles. Trondheim, Norway: Marine Cybernetics; 2002.

[4] N. Wang, M. Han, N. Dong, and M. J. Er, "Constructive multioutput extreme learning machine with application to large tanker motion dynamics identification," Neurocomputing, vol. 128, pp. 59-72, Mar. 2014.

[5] X. Xiang, C. Yu, and Q. Zhang, "Robust fuzzy 3D path following for autonomous underwater vehicle subject to uncertainties," Comput. Oper. Res., to be published.

[6] R. Cui, X. Zhang, and D. Cui, "Adaptive sliding-mode attitude control for autonomous underwater vehicles with input nonlinearities," Ocean Eng., vol. 123, pp. 45-54, 2016.

[7] X. Xiang, L. Lapierre, and B. Jouvencel, "Smooth transition of AUV motion control: From fully-actuated to under-actuated configuration," Robot. Auton. Syst., vol. 67, no. 5, pp. 14-22, Mar. 2015.

[8] S. Serdar, J. B. Bradley, and P. P. Ron, "A chattering-free sliding-mode controller for underwater vehicles with fault-tolerant infinity-norm thrust allocation," Ocean Engineering, vol. 35, no. 16, pp. 1647-1659, Nov. 2008.

[9] Y. Yang, J. L. Du, H. B. Liu, C. Guo, and A. Abraham, "A trajectory tracking robust controller of surface vessels with disturbance uncertainties," IEEE Trans. Control Syst. Technol., vol. 22, no. 4, pp. 1511-1518, Jul. 2014.

[10] K. Y. Pettersen, and H. Nijmeijer, "Tracking control of an underactuated surface vessel," In: Proceedings of the 37th IEEE Conference on Decision and Control, pp. 4561-4566, 1998.
[11] K. Shojaei, "Neural adaptive robust control of underactuated marine surface vehicles with input saturation," Appl. Ocean Res., vol. 53, pp. 267-278, Sep. 2015.

[12] G. Q. Zhang, and X. K. Zhang, "Concise robust adaptive path-following control of underactuated ships using DSC and MLP," IEEE J. Ocean Eng., vol. 39, no. 4, pp. 685-694, Oct. 2014.

[13] Z. Zhao, W. He, and S. S. Ge, "Adaptive neural network control of a fully actuated marine surface vessel with multiple output constraints," IEEE Trans. Control Syst. Technol., vol. 22, no. 4, pp. 1536-1543, Jul. 2014.

[14] Z. Yan, and J. Wang, "Model predictive control for tracking of underactuated vessels based on recurrent neural networks," IEEE J. Ocean Eng., vol. 37, no. 4, pp. 717-726, Oct. 2012.

[15] H. Li, P. Shi, and D. Yao, "Adaptive sliding mode control of Markov jump nonlinear systems with actuator faults," IEEE Trans. Automat. Contr., to be published.

[16] H. Li, C. Wu, X. Jing, and L. Wu, "Fuzzy tracking control for nonlinear networked systems," IEEE Trans. Cybern., to be published.

[17] Y. M. Li, S. C. Tong, and T. S. Li, "Adaptive fuzzy output feedback dynamic surface control of interconnected nonlinear pure-feedback systems," IEEE Trans. Cybern., vol.45, no.1, pp.138-149, Jan. 2015.

[18] Y. M. Li, S. C. Tong, and T. S. Li, "Composite adaptive fuzzy output feedback control design for uncertain nonlinear strict-feedback systems with input saturation," IEEE Trans. Cybern., vol. 45, no. 10, pp. 22992308, Oct. 2015.

[19] B. Xu, F. C. Sun, Y. P. Pan, and B. D. Chen, "Disturbance observer based composite learning fuzzy control of nonlinear systems with unknown dead zone," IEEE Trans. Syst., Man, Cybern., Syst., to be published.

[20] C. S. Chen, "Dynamic structure neural-fuzzy networks for robust adaptive control of robot manipulators," IEEE Trans. Ind. Electron., vol. 55, no. 9, pp. 3402-3414, Sep. 2008.

[21] N. Wang, and M. J. Er, "Self-constructing adaptive robust fuzzy neural tracking control of surface vehicles with uncertainties and unknown disturbances," IEEE Trans. Control Syst. Technol., vol. 23, no. 3, pp. 991-1002, May 2015.

[22] A. Wu, and P. K. Tam, "Stable fuzzy neural tracking control of a class of unknown nonlinear systems based on fuzzy hierarchy error approach," IEEE Trans. Fuzzy Syst., vol. 10, no. 6, pp. 779-789, Dec. 2002.

[23] W. He, Y. H. Chen, and Z. Yin, "Adaptive neural network control of an uncertain robot with full-state constraints," IEEE Trans. Cybern., vol. 46, no. 3, pp. 620-629, Mar. 2016.

[24] W. He, Y. Dong, and C. Sun, "Adaptive neural impedance control of a robotic manipulator with input saturation," IEEE Trans. Syst. Man Cybern.: Syst., vol. 46, no. 3, pp. 334-344, Mar. 2016.

[25] S. I. Han, and J. M. Lee, "Recurrent fuzzy neural network backstepping control for the prescribed output tracking performance of nonlinear dynamic systems," ISA Trans., vol. 53, no. 1, pp. 33-43, Jan. 2014.

[26] N. Wang, M. J. Er, J. C. Sun, and Y. C. Liu, "Adaptive robust online constructive fuzzy control of a complex surface vehicle system," IEEE Trans. Cybern., vol. 46, no. 7, pp. 1511-1523, Jul. 2016.

[27] N. Wang, J. C. Sun, M. J. Er, and Y. C. Liu, "A novel extreme learning control framework of unmanned surface vehicles," IEEE Trans. Cybern., vol. 46, no. 5, pp. 1106-1117, May 2016.

[28] N. Wang, and M. J. Er, "Direct adaptive fuzzy tracking control of marine vehicles with fully unknown parametric dynamics and uncertainties," IEEE Trans. Control Syst. Technol., vol. 24, no. 5, pp. 1845-1852, Sep. 2016.

[29] N. Wang, M. J. Er, and M. Han, "Dynamic tanker steering control using generalized ellipsoidal-basis-function-based fuzzy neural networks," IEEE Trans. Fuzzy Syst., vol. 23, no. 5, pp. 1414-1427, Oct. 2015.

[30] K. F. Lu, Y. Q. Xia, Z. Zhu, and M. V. Basin, "Sliding mode attitude tracking of rigid spacecraft with disturbances," J. Franklin Institute, vol. 349, no. 2, pp. 423-440, Mar. 2012.

[31] Y. G. Hong, Y. S. Xu, and H. Jie, "Finite-time control for robot manipulators," Syst. Control Lett., vol. 46, pp. 243-253, Jan. 2002.

[32] W.-H. Chen, D. J. Ballance, P. J. Gawthrop, and J. O'Reilly, "A nonlinear disturbance observer for robotic manipulators," IEEE Trans. Ind. Electron., vol. 47, no. 4, pp. 932-938, Aug. 2000.

[33] J. Yang, W.-H. Chen, and S. Li, "Robust autopilot design of uncertain bank-to-turn missiles using state-space disturbance observers," Proc. Inst. Mech. Eng., J. Aerosp. Eng., vol. 226, no. 1, pp. 97-107, Jan. 2012.

[34] W.-H. Chen, K. Ohnishi, and L. Guo, "Advances in disturbance/uncertainty estimation and attenuation," IEEE Trans. Ind. Electron., vol. 62, no. 9, pp. 5758-5762, Sep. 2015.

[35] W.-H. Chen, J. Yang, L. Guo, and S. H. Li, "Disturbance-observer-based control and related methods-an overview," IEEE Trans. Ind. Electron., vol. 63, no. 2, pp. 1083-1095, Sep. 2015. 
[36] T. Umeno, and Y. Hori, "Robust speed control of dc servomotors using modern two degrees-of-freedom controller design," IEEE Trans. Ind. Electron., vol. 38, no. 5, pp. 363-368, Oct. 1991.

[37] W.-H. Chen, "Nonlinear disturbance observer-enhanced dynamic inversion control of missiles," J. Guid. Control Dyn., vol. 26, no. 1, pp. 161166, Jan. 2003.

[38] J. Yang, S. H. Li, X. S. Chen, and Q. Li, "Disturbance rejection of deadtime processes using disturbance observer and model predictive control," Chem. Eng. Res. Des., vol. 89, no. 2, pp. 125-135, Feb. 2011.

[39] H. J. Marquez, Nonlinear Control Systems: Analysis and Design, John Wiley \& Sons, Inc Hoboken, New Jersey, 2003.

[40] H. Khalil, Nonlinear Systems, 2nd ed. Upper Saddle River, NJ: PrenticeHall, 1996.

[41] A. Levant, "Homogeneity approach to high-order sliding mode design," Automatica, vol. 41, no. 5, pp. 823-830, May 2005.

[42] Y. B. Shtessel, I. A. Shkolnikov, and A. Levant, "Smooth second-order sliding modes: Missile guidance application," Automatica, vol. 43, no. 8 , pp. 1470-1476, Aug. 2007.

[43] R. Skjetne, T. I. Fossen, and P. V. Kokotović, "Adaptive maneuvering, with experiments, for a model ship in a marine control laboratory," Automatica, vol. 41, no. 2, pp. 289-298, Feb. 2005.

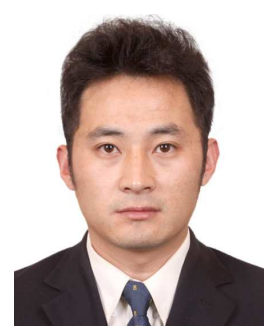

Ning Wang (S'08-M'12-SM'15) received his B. Eng. degree in Marine Engineering and the Ph.D. degree in control theory and engineering from the Dalian Maritime University (DMU), Dalian, China in 2004 and 2009, respectively. From September 2008 to September 2009, he was financially supported by China Scholarship Council (CSC) to work as a joint-training $\mathrm{PhD}$ student at the Nanyang Technological University (NTU), Singapore. In the light of his significant research at NTU, he received the Excellent Government-funded Scholars and Students Award in 2009. From August 2014 to August 2015, he worked as a Visiting Scholar at the University of Texas at San Antonio. He is currently a Full Professor with the Marine Engineering College, DMU, Dalian 116026, China.

Dr. Wang received the Nomination Award of Liaoning Province Excellent Doctoral Dissertation, the DMU Excellent Doctoral Dissertation Award and the DMU Outstanding PhD Student Award in 2010, respectively. He also won the Liaoning Province Award for Technological Invention and the honour of Liaoning BaiQianWan Talents, Liaoning Excellent Talents, Science and Technology Talents the Ministry of Transport of the P. R. China, Youth Science and Technology Award of China Institute of Navigation, and Dalian Leading Talents. His research interests include fuzzy neural systems, deep learning, nonlinear control, self-organizing fuzzy neural modeling and control, unmanned vehicles and autonomous control. He currently serves as Associate Editors of the Neurocomputing and the International Journal of Fuzzy Systems.

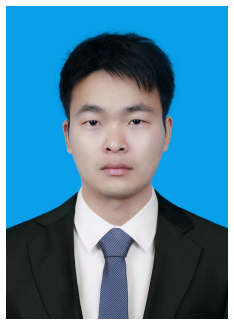

Shuailin $\mathbf{L v}$ received his B. Eng. degree in Marine Electronic and Electrical Engineering and B. E. degree in International Economics and Trade from the Dalian Maritime University (DMU), China in 2015. He is currently pursuing his Master degree at the same university. His research interests include electric drive and control, sliding mode control, marine control, and finite-time control.

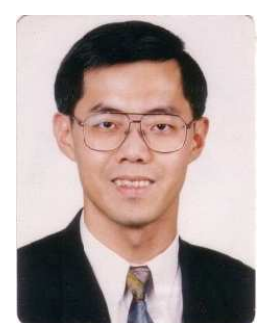

Meng Joo Er (S'82-M'87-SM'07) is currently a Full Professor in Electrical and Electronic Engineering, Nanyang Technological University, Singapore. He has authored 5 books, 16 book chapters and more than 500 refereed journal and conference papers in his research areas of interest. His areas of research interests are intelligent control theory and applications, computational intelligence, robotics and automation, sensor networks, biomedical engineering and cognitive science.

In recognition of the significant and impactful contributions to Singapores development by his research project entitled Development of Intelligent Techniques for Modelling, Controlling and Optimizing Complex Manufacturing Systems, Professor Er won the Institution of Engineers, Singapore (IES) Prestigious Engineering Achievement Award 2011. He is also the only dual winner in Singapore IES Prestigious Publication Award in Application (1996) and IES Prestigious Publication Award in Theory (2001). He received the Teacher of the Year Award for the School of EEE in 1999, School of EEE Year 2 Teaching Excellence Award in 2008 and the Most Zealous Professor of the Year Award 2009. He also received the Best Session Presentation Award at the World Congress on Computational Intelligence in 2006 and the Best Presentation Award at the International Symposium on Extreme Learning Machine 2012. Under his leadership as Chairman of the IEEE CIS Singapore Chapter from 2009 to 2011, the Singapore Chapter won the CIS Outstanding Chapter Award 2012. In recognition of his outstanding contributions to professional bodies, he was bestowed the IEEE Outstanding Volunteer Award (Singapore Section) and the IES Silver Medal in 2011. On top of this, he has more than 40 awards at international and local competitions. Currently, Professor Er serves as the Editor-in-Chief of the Transaction on Machine Learning and Artificial Intelligence, an Area Editor of International Journal of Intelligent Systems Science, an Associate Editor of eleven refereed international journals including the IEEE Transaction on Fuzzy Systems and IEEE Transactions on Cybernetics, and an editorial board member of the EE Times. He has been invited to deliver more than 60 keynote speeches and invited talks overseas.

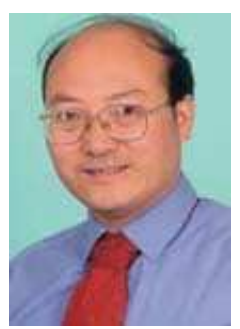

Wen-Hua Chen (SM'06) received the M.Sc. and $\mathrm{Ph} . \mathrm{D}$. degrees from Northeast University, Shenyang, China, in 1989 and 1991, respectively.

From 1991 to 1996, he was a Lecturer and then Associate Professor with the Department of Automatic Control, Nanjing University of Aeronautics and Astronautics, Nanjing, China. From 1997 to 2000, he held a research position and then was a Lecturer of control engineering with the Centre for Systems and Control, University of Glasgow, Glasgow, U.K. In 2000, he moved to the Department of Aeronautical and Automotive Engineering, Loughborough University, Loughborough, U.K., as a Lecturer, where he was appointed as a Professor in 2012. His research interests include the development of advanced control strategies (nonlinear model predictive control, disturbance-observer-based control, etc.) and their applications in aerospace and automotive engineering. Currently, much of his work has also been involved with the development of unmanned autonomous intelligent systems.

Dr. Chen is a Fellow of The Institution of Engineering and Technology, U.K., and a Fellow of The Institution of Mechanical Engineers, U.K. 